\title{
Surface Roughness Evolution During Early Stages of Mechanical Cyclic Loading
}

\author{
Ahmed M. Hussein, Jaafar A. El-Awady \\ Department of Mechanical Engineering \\ Johns Hopkins University, Baltimore, MD 21218-2681, USA
}

\begin{abstract}
The effect of crystal size and initial dislocation density on surface roughness evolution in FCC single crystals during the early number of cycles of mechanical cyclic loading is investigated using three dimensional discrete dislocation dynamics simulations. Crystals having size less than $2 \mu \mathrm{m}$ show early development of surface slip localization, while larger ones show a more uniform distribution of surface steps. The surface roughness is found to increase with increasing number of loading cycles with larger crystals showing a high roughening rate compared to smaller crystals. Double cross-slip is observed to be the main mechanism that derives the development, growth and thickening of surface slip bands. The maximum surface height, which is an indicator of the surface stress concentration is observed to increase linearly with the number of loading cycles and quadtratically with the crystal size for the simulated number of cycles. Finally, the results are shown to be in agreement with experimental results and provide further physics based understanding on the mechanisms controlling the evolution of the surface roughness.
\end{abstract}

Keywords:

Surface Roughness, Cyclic Loading, Dislocation Dynamics, Cross-slip, Hausdorff Dimension

\section{Introduction}

Fatigue under cyclic loading is one of the leading causes of failure in mechanical and structural components $[1,2]$. While fatigue failure has been subject to intensive studies

Email addresses: ahussei4@jhu.edu (Ahmed M. Hussein), jelawady@jhu.edu (Jaafar A. El-Awady) Preprint submitted to Int. J. Fatigue

February 7, 2016 
for almost 150 years $[3,4]$, a complete understanding of the complex mechanisms that lead to fatigue failure is still elusive [3]. The complexity of the fatigue failure process stems from its sensitivity to many factors, including the loading type, microstructure, material heterogeneity, and environmental conditions among other factors [5]. Furthermore, the mechanisms responsible for fatigue crack initiation and propagation take place over multiple length and time scales [6].

The fatigue failure process can be divided into two stages, fatigue crack initiation (nucleation), and fatigue crack propagation $[4,5]$. The crack nucleation stage is the least understood of the two and understanding the mechanisms that control it is the first step in the process of extending the lifespan of materials through appropriate microstructure design. Cracks can nucleate at grain/twin boundaries [7], at an inclusion interface [8], or at the crystal free surface $[5,6,9]$, with the latter being more prominent at room temperature $[5,9]$. One of the earliest experimental observations of what was understood later as a precursor to fatigue crack nucleation at a free surface was made by Ewing et. al. [10], where the surface was observed to roughen in the form of sharp surface slip bands on the surface of polycrystalline iron specimens. These surface slip bands are in the form of extrusions/intrusions that render an initially smooth crystal surface into a rough one, and the heights/depths of these extrusions/intrusions were shown to increase with increasing number of cycles $[4,11,12]$. The edges of these extrusions/intrusions usually make sharp angles with the crystal surface leading to high local stress concentrations. When these local stresses are high enough, atomic bonds break leading to surface crack nucleation [13, 14]. This view was further confirmed by a number of experimental studies $[6,15,16,17,18,19,20]$.

With the growing interest in micro/nanoelectronics and micro-electrical-mechanical systems, significant interest has been directed towards developing an understanding of the mechanical properties of metals at small scales. While numerous experimental and computational studies have focused on size-scale effects on the mechanical properties of metals during monotonic loading (c.f. [21, 22]), far less attention has been directed towards understanding the effect of the crystal size on the cyclic response of microcrystals. It should be noted that experimental studies of the cyclic deformation of microcrystals is technologically 
more challenging than bulk crystal studies. Nevertheless, a number of recent experimental studies have focused on the response of cantilever microbeams under cyclic bending [23, 24] as well as microwires under cyclic torsion [25, 26] loadings. However, none of these studies have focused on the effect of crystal size or initial dislocation density on the surface roughness evolution in microcrystals, which is important to understand as a precursor to crack initiation.

Over the past two decades, discrete dislocation dynamics (DDD) simulations have been extensively used to study many phenomena in the plastic deformation of metals [27]. However, only few DDD studies focused on simulations of cyclic loading. Of these, two-dimensional (2D) DDD simulations of dislocation interactions with pre-existing cracks during monotonic and cyclic loading suggested that the dislocation density multiplication and plastic-zone size near the crack-tip are weakly influenced by the crack size [28]. Moreover, three-dimensional (3D) DDD simulations of a single surface grain during early stages of mechanical cyclic loading showed that the height of surface steps that form by dislocation escape from the free surface increases with increasing number of loading cycles and imposed plastic strain amplitude $[29,30]$. However, those 3D DDD simulations were performed starting with a single Frank-Read source in the simulation cell, which corresponds to an unrealistically low initial dislocation density. In addition, 3D DDD simulations were also conducted to study the effect of long range stresses on the dynamics of screw dislocations in a PSB channel and the PSB flow strength [31]. Finally, the current authors performed a large set of 3D DDD simulations to quantify the dislocation microstructure evolution and cyclic hardening in free standing single crystals [32]. These simulations showed that the crystal size plays the dominant role in controlling dislocation multiplication, formation of dislocation cell-like structures, and cyclic hardening.

While such DDD studies have shed light on some aspects of cyclic loading, many important open questions remain to be addressed, including the role of crystal size and initial dislocation density on the surface roughness evolution and surface slip localization. To this end, the current work is an attempt in that direction. The paper is organized as follows. In Section 2, the computational methods are discussed, while the details of the numerical 
simulations are given in Section 3. In Section 4, the simulations results are presented and discussed in terms of the active dislocation mechanisms in an attempt to correlate the surface roughness evolution and the dislocation microstructure evolution. Finally, in Section 5 a summary and conclusions are presented.

\section{Computational Methods}

All simulations results presented here are performed using a version of the open source 3D DDD code ParaDiS [33] that has been extensively modified by the authors at Johns Hopkins University. The original ParaDis structure was retained, however, the dislocation mobility routine for face-centered-cubic (FCC) crystals was altered to enforce the dislocation glide on the proper $\{111\}$ slip planes, eliminating any possibility for artificial or unphysical dislocation climb. In addition, the algorithms handling short range interactions (e.g. dislocation collisions and topological operations) were redesigned to prevent the erroneous reorientation of dislocation segments out of their slip planes. Furthermore, a new atomistically-based cross-slip model has been implemented in the code [34]. This model accounts for the three recently characterized cross-slip mechanisms known as bulk, intersection, and surface crossslip [35, 36, 37, 38, 39].

New rules for tracking dislocation interactions with free surfaces were implemented to compute the surface displacement fields and model surface roughness evolution. When a dislocation segment escapes from the crystal free surface it is removed from the pool of active dislocations, and the time, location, and slip plane of the escape event are stored. In large simulations having large dislocation densities, the continuous storage of the records of escaping dislocation segments can lead to memory issues, as well as significant slow down in the displacement field calculations. Thus, a reduction algorithm was implemented to compress the escaped segment records when possible. The reduction is a lossless compression algorithm, in which the surface displacements due to the original dislocation-surface interaction information can be fully recovered from the compressed data. In the following two subsections, the displacement field calculations and the quantification of the surface roughness are discussed in details. 


\subsection{Displacement Field Calculation}

The closed form solution for the displacement field of a single dislocation loop in an isotropic medium can be expressed as a line integral as follows [40]:

$$
u_{i}=-\frac{b_{i} \Omega}{4 \pi}+\frac{1}{8 \pi} \oint_{C}\left(\epsilon_{i k l} b_{l} r_{, p p}+\frac{1}{1-\nu} \epsilon_{k m n} b_{n} r_{, m i}\right) d l_{k}
$$

where $u_{i}(i=1,2$, and 3$)$ are the three displacement components of the displacement vector, $\Omega$ is the solid angle subtended by the dislocation loop at the field point at which the displacement is calculated, $b_{i}$ are the components of the dislocation's Burger's vector, $C$ is the dislocation loop curve, $\epsilon_{i j k}$ are the components of the permutation tensor, $\nu$ is the material's Poisson's ratio, and $r$ is the magnitude of the position vector between a point on the dislocation curve and the field point.

The continuous evaluation of the displacement field as defined by Equation 1 necessitates the tracking of each dislocation loop throughout the simulation. To alleviate the significant computational burden associated with this continuous tracking of all evolving dislocation loops during the simulation, especially in the case of non-planar loops that continuously evolve on new slip planes due to cross-slip, a more efficient algorithm is developed here. In this algorithm, a virtual dislocation loop is created for each dislocation segment under consideration. As shown schematically in Figure 1, for any dislocation segment $(A B)$ that escapes the free surface, an equivalent 5-segment closed-loop $(O A C D B O)$ is created during the displacement calculation step. This equivalent loop is constructed by first choosing an arbitrary anchor point, $O$ inside the simulation volume. In the current simulations this point is chosen to be the point of intersection of the segment's slip plane with the z-axis. A copy $(C D)$ of the dislocation segment $(A B)$ is then created parallel to it on the same slip plane at a distance $d$ such that both vectors $(A C)$ and $(B D)$ are normal to the crystal surface and lie on the same slip plane. The accuracy of the displacement calculations increases with increasing $d$ [41]. It should be noted that this equivalent loop is planar and lies on the slip plane of the original dislocation segment. Consequently, when generating the equivalent loops for all the surface dislocation segments, the displacement fields from the $(O A)$ 
segment of one loop and $(B O)$ of the adjacent loop cancel out. Similarly, segments $(A C)$ and $(D B)$ of two adjacent loops cancel each other out. Hence, the end result of the displacement calculation is the displacement due to the actual surface dislocations without any contribution from the added equivalent dislocation loop segments. This makes the process of calculating the displacement fields of planar and non-planar loops straightforward and accurate with no further special handling required, unlike other proposed approaches [42]. In these calculations, the displacement field of any dislocation segment not intersecting the surface is neglected since this field is smooth on the crystal surface and does not contribute to abrupt changes in the surface profile (i.e. surface roughness).

On the other hand, the solid angle term is typically computed as an equivalent closedloop integration [43]. However, this formulation requires the selection of an arbitrary vector that can result in non-unique displacement fields under certain conditions. Alternatively, the solid angle of a geometric object subtended at a point $P$ is defined as the area of the projection of the object on a unit sphere centered at $P$. Thus, the solid angle term in Equation (1) is computed here by triangulating the planar equivalent dislocation loop and then the individual triangles are projected on a unit sphere centered at the field point. The area of the projected triangles can be calculated and summed giving the exact value for the solid angle. It is worth noting that the surface dislocation tracking method described here and the subsequent surface displacement calculations apply as is for all convex simulation cell geometries such as cylindrical and dodecahedral cells.

\subsection{Surface Roughness Quantification}

One typical way to quantify surface roughness is by using the maximum peak height, the minimum valley depth, and the first few statistical moments of the displacement amplitude distribution [44]. These parameters are typically used to correlate surface roughness to crack nucleation and failure events [5]. However, the dislocation evolution is usually heterogeneous, which leads to non-uniform dislocation surface escape events.

In order to correlate the surface roughness to the dislocation microstructure evolution, a roughness measure that takes into account the displacement amplitudes as well as their 
spatial distribution is required. One such measure is the Hausdorff dimension [45, 46], which is a measure of the local surface complexity. The Hausdorff dimension is calculated by discretizing the undeformed surface into a uniform squared mesh with an element edge length $\epsilon$. The surface displacement field is then interpolated at the corners of each element and the deformed surface geometry is obtained. The total area, $A$, of the deformed surface is then computed by summing the areas of each deformed element. This calculation is repeated for different values of $\epsilon$, then the $\epsilon-A$ relationship is fitted to a power law having the form

$$
A(\epsilon)=A_{o} \epsilon^{2-D}
$$

where $A_{o}$ is the area of the undeformed surface, and $D$ is the Hausdorff dimension, which ranges between 2 for a perfectly smooth surface to 3 for an infinitely rough surface.

The Hausdorff dimension is directly related to the slope of the power spectral density (PSD) distribution curve [47, 48], which can also be used as a roughness measure. The PSD distribution gives the power in the normal displacement signal as a function of the normal displacement wavelength (or frequency). The surface roughness increases when higher powers are associated with higher frequencies and vice versa. The advantage of using the Hausdorff dimension over the PSD is that the Hausdorff dimension is a single scalar that quantifies the roughness distribution over the surface while the PSD is a one dimensional distribution.

\section{Numerical Simulations}

All simulations performed here assume material properties of face-centered cubic (FCC) nickel (Young's modulus $E=210 \mathrm{GPa}$, and Poisson's ratio $\nu=0.31$ ). The simulation cells are considered as free standing single crystals having cubic geometries with side lengths varying between $0.5 \leq D \leq 5.0 \mu \mathrm{m}$. The edges of the simulation cell are parallel to the $\langle 100\rangle$ directions, as shown schematically in Figure 2. The six faces of the simulation cell are modeled as free surfaces and the loading is imposed uniformly on the top and bottom surfaces of the crystal oppositely in the [001] direction. The loading is a fully reversed total 
strain controlled mechanical cyclic loading under a triangular wave loading profile with a constant total strain rate of $\dot{\epsilon}=200 \mathrm{~s}^{-1}$, and a total strain amplitude of $\Delta \varepsilon=0.4 \%$. Thus, these situations mimic an experimental setting where the crystal is free standing and it is supported from both ends by soft grips. The initial dislocation density is varied from $\rho_{o}=10^{11}$ to $10^{14} \mathrm{~m}^{-2}$ and the initial dislocation microstructure consists of a random distribution of of FR sources having lengths normally distributed with a mean length of $0.6 D$ and a standard deviation of $0.03 D$. The FR sources are randomly distributed inside the crystal volume with uniform probability over all twelve FCC slip systems to reproduce a realistic dislocation microstructure evolution. Each simulation of a given crystal size and initial dislocation density is repeated three times with a different random starting FR source distribution to capture any statistical variations due to the initial dislocation distribution. In all current simulations, the image forces due to the free surfaces are neglected since they have a minimal effect on the overall dislocation microstructure evolution in crystals having $D \geq 0.5 \mu \mathrm{m}[30,49]$. Finally, all possible cross-slip mechanisms are allowed in all simulations with the appropriate activation energies and volumes reported in [34].

\section{Results and Discussion}

The size effect on cyclic hardening, dislocation multiplication rates, and dislocation structure formation were fully discussed in a previous publication by the authors [32], and is beyond the scope of the current study. Nevertheless, for completeness a short synopsis is presented here first. Representative curves of the engineering stress and dislocation density versus the simulation time for simulations with an initial dislocation density of $\rho_{0}=10^{-12}$ $\mathrm{m}^{-2}$ and crystal sizes $D=0.5,0.75,1.0,2.0,5.0$, and $7.5 \mu \mathrm{m}$ are shown in Figure 3. In all simulations of different initial dislocation densities, it was shown that the cyclic hardening and dislocation density multiplication rate increase with increasing crystal size. Furthermore, the dislocation microstructure was shown to arrange in cell-like structures with high dislocation density walls and low dislocation density interiors only in crystals having sizes $D \geq 2.0 \mu \mathrm{m}$. The cell size and the wall thicknesses were shown to be independent of the number of cycles for the number of cycles modeled, while their numbers increase with in- 
creasing crystal size. Detailed discussions regarding the mechanical response and dislocation microstructure evolution can be found in [32]. In the following the focus is dedicated towards quantifying the evolution of the surface roughness as predicted from these simulations.

Figure 4 shows the surface morphology after 5 loading cycles for four representative simulations having different edge lengths and initial dislocation densities. In addition, the surface roughness evolution of a $D=0.75 \mu \mathrm{m}$ simulation cell having an initial dislocation density of $\rho_{o}=10^{12} \mathrm{~m}^{-2}$ is shown in supplementary movie I. The major steps on the surface correspond to slip planes where the dislocation activity is the highest. The minor steps parallel to the major ones form due to double cross-slip events, which lead to the introduction of dislocations on parallel slip planes. On some surfaces, especially for smaller crystals when few dislocations are active, major surface steps make an angle of $45^{\circ}$ with each other. This is a sign of a single cross-slip event introducing dislocations on the cross-slip planes of the active dislocation sources since FCC slip planes intersect the surface $\{001\}$ planes at lines making an angle of $45^{\circ}$ with the principal axes. When the surface plane contains the Burgers vector of a family of dislocations, no observable surface roughening takes place although the surface atoms are still displaced within their plane. The displacement contours are shown in Figure 5 for the top surfaces of the same crystals shown in Figure 4 at the same number of loading cycles where the $45^{\circ}$ angle between the active slip plane is also evident. One can also qualitatively observe that the surface displacement magnitude scales with the crystal size. A more solid quantitative treatment of this observation is discussed below.

Two main modes of surface roughness can be qualitatively identified from these simulations. In the first mode, most plasticity is localized in a few deformation bands as shown in Figure 4(d). In this case, surface steps form and evolve contiguously within these bands with the remainder of the surface being mostly "smooth". This is generally observed in simulations of smaller crystal sizes and/or in simulations starting with a low initial dislocation density. In the second mode, slip is distributed uniformly over the entire surface of the crystal as shown in Figure 4(a). In addition, distinct steps that are higher than their surroundings are also observed. This usually is observed in simulations of larger crystal sizes, or when the initial dislocation density is high. It can also be argued that the uniform 
roughness distribution takes place at early cycles, while localization in surface slip bands takes place later as has been observed in earlier studies [30]. The evolution of the surface displacements of crystals having the same initial dislocation density of $\rho_{o}=10^{12} \mathrm{~m}^{-2}$ and different sizes for different number of loading cycles is shown in Figure 6 where the spread of the surface roughness can be observed to take place for the larger crystal at a rate higher than that of the smaller crystal.

As shown schematically in Figure 7, the formation, growth, and thickening of surface slip bands can be correlated with the surface cross-slip activity. Surface cross-slip is the most frequent since it has the lowest activation energy [34], thus, dislocation segments terminating on the surface frequently engage in surface cross-slip events that take one dislocation from one glide plane, and through double cross-slip, put it back on a plane parallel to its original glide plane. Combined with cyclic loading, the dislocation glides in one direction on its slip plane and in the opposite direction after double cross-slip, which causes the volume between the two planes to gradually extrude (or intrude). Furthermore, at the beginning of the surface slip band formation, the dislocation traverse distance is almost purely stochastic and the second cross-slip event can occur anywhere. However, as the array of dislocations on parallel slip planes gets denser, the stresses from the dislocations gliding on parallel planes can reduce the surface cross-slip activation energy further. This is because the activation energy of cross-slip depends on the separation distance of the two dislocation partials, which in turn depends on the Escaig stress acting on them (the Escaig stress is the stress component that brings the two partials closer or separates them further apart depending on its sign [34]). Thus, the local stress field is an influencing factor on the cross-slip probability, and dislocations would preferentially cross-slip on unoccupied parallel planes where the back stresses are relatively lower.

Figure 8 shows both the surface roughness on two opposite crystal surfaces as well as the internal dislocation microstructure after different loading cycles for a crystal having edge length $D=2.0 \mu \mathrm{m}$ and an initial dislocation density of $\rho_{o}=10^{13} \mathrm{~m}^{-2}$. It is clear that the dislocation density near the free surface is distinctly lower than in the interior. This is due to the high degree of dislocation out-flux from the crystal surface as opposed to the 
high degree of dislocation storage in the interior of the crystal where the thick dislocations entanglements that form block other dislocations from motion. This would correspond to a lower stress near the surface, and also implies that the surface region would experience more plastic strain than the interior during every loading cycle [50]. These results are in agreement with monotonically loaded and cyclic loaded thin copper single crystals, in which it was shown that a flow stress gradient develops between the crystal surface and center $[51,50,52]$.

Furthermore, surface steps form initially near the middle of the surface and spreads out with increasing number of loading cycles. A similar trend can be observed for the dislocation microstructure, which becomes more dense as the number of cycles increases. In addition, the heights of the surface steps increase with increasing number of loading cycles, which implies that the developed roughness is irreversible even though the applied load is. The strong correlation between surface roughness and dislocation microstructure evolution as well as the surface roughness irreversibility have been observed in all simulated cases. While the former observation is expected, the latter needs further explanation. For a step to be erased, a dislocation with a Burgers vector opposite to that of the step has to be deposited at the exact location of the original step. The load reversal guarantees that these dislocations exist even in the cases of very low densities (in fact, a single Frank-Read source loaded cyclically would deposit both positive and negative steps on the surface), however, cross-slip usually moves the "erasing" dislocations to parallel planes before they get a chance to cancel out the already formed steps since the time-scale at which cross-slip operates is much smaller than one half of a loading period. This explains the emergence of regions on the surface where the normal surface displacement rapidly fluctuates (i.e. regions where extrusions and intrusions form right next to each other on parallel planes).

The first few statistical moments (e.g. the mean and standard deviation) of the surface height distribution can be used to describe the evolution of the surface topography over time. However, crack nucleation depends on the stress concentration, which increases with increasing step height/depth. Thus, the most critical surface feature that should be tracked is the maximum surface height (MSH), defined here as the difference between the height 
of the highest surface peak and the depth of the deepest surface valley with the peaks and valleys determined based on the displacement field normal to the surface. The variations of MSH versus the number of loading cycles for different crystal sizes and initial dislocation densities are shown in Figures 9 and 10, respectively. In all simulated cases, there is a persistent growth in MSH as previously qualitatively discussed based on the deformed surface visualizations in Figure 4, and it is observed to increase with increasing cycle number. The MSH growth follows a staircase pattern in most cases with a burst in the step formation followed by a longer time interval with no new step development. The bursts take place slightly after the beginning of each cycle, and they correspond to the end of the elastic loading. This is the time when the applied stress reaches its peak value right before yielding, which suggests that the applied stress is the main driver for surface step formation rather than the dislocation interaction stresses. Furthermore, while not plotted here, the rate of growth of the mean absolute height, defined as the average of the peak heights and valley depths, is predicted from the current simulations in the range of $0.01 \mathrm{~nm} /$ cycle to 0.45 $\mathrm{nm} /$ cycle, which is in good agreement with experimental measurements in the first few hundred cycles [18].

Figures 11(a) shows the variation of the MSH versus crystal size from all simulations having $\rho_{o}=10^{12} \mathrm{~m}^{-2}$ at different number of loading cycles. It is observed that MSH increases with increasing crystal size, and its increase with increasing number of cycles, $N$, is more rapid for larger crystals. By fitting the data to a power law it is observed that the MSH for any given cycle number correlates with the square of the edge length (i.e. the surface area) of the simulation volume, with a correlation coefficient exceeding 0.76. Furthermore, the data also indicate that the MSH is proportional to the number of loading cycles for the number of cycles modeled here. Thus, it it can be shown that for $\rho_{o}=10^{12}$ $\mathrm{m}^{-2}$ the MSH follows the relationship:

$$
h_{\max }=0.78 N D^{2}
$$

where $h_{\max }$ is the MSH. The linear variation with $N$ implies that the dislocation flux from 
the crystal surface is constant such that the number of dislocations (or more precisely, the total Burgers vector) leaving the surface per unit time does not change for the same initial dislocation density. The quadratic variation with $D$ is due to the number of sources that can contribute to a particular surface peak/valley. In FCC materials at room temperatures the dislocations move mainly by dislocation glide [53, 54], hence, all dislocations escaping at a particular location on the surface glide on a particular slip plane that intersects the surface at this location. For a dislocation to glide on this particular plane, it needs to either be emitted from a dislocation source on this plane, or to be a result of a cross-slip event onto it. For a constant initial dislocation density, the number of sources per slip plane increases with increasing plane area, which is proportional to $D^{2}$. Similarly, for a homogeneous stress field and temperature, the probability of cross-slip onto a particular plane increases with the plane's area [34]. It should be noted that this correlation is strictly valid for the first few hundred loading cycles. As was shown experimentally the step heights can increase nonlinearly with increasing number of cycles after the first 1000 cycles [18], which is beyond the scope of the current simulations.

Figure 11(b) shows the variation of MSH with the initial dislocation density for three crystal sizes after 5 loading cycles. In the simulations of large crystal sizes, or those starting with high initial dislocation densities, the dislocation density multiplication rate is higher [32], which in turn leads to a fast increase in the number of degrees of freedom. Thus, the simulations starting with an initial dislocation density $\rho_{o} \geq 10^{12} \mathrm{~m}^{-2}$ where only continued for less than 10 cycles. As such, it is difficult based on the limited number of cycles reached in these simulations to derive a correlation between MSH, size, initial dislocation density, and number of cycles. Nevertheless, Figure 11(b) clearly shows a power law correlation between MSE and the initial dislocation density at a given crystal size and number of cycles. Further simulations are still needed to be able to derive a more general correlation.

The variation of the Hausdorff dimension with the number of loading cycles for different crystal sizes and initial dislocation densities is shown in Figures 12 and 13, respectively. Certain features are similar to those observed from the MSH evolution curves including the monotonic increase in the roughening rate as well as the direct relationship between the 
roughening rate and the crystal size. The drops in the HSD curves correspond to the partial removal of a surface step by a dislocation of an opposite sign, which reduces the overall step height. However, this does not necessarily reduce the surface roughness. The only way by which the surface roughness, and hence, the HSD would decrease is by the complete removal of a surface step such that the surface can return locally to its undeformed configuration. Practically, this should be an extremely rare event since it requires the exact number of oppositely signed dislocations escaping right at this surface step causing it to recede (or advance for intrusions) and vanish. Finally, the correlation between HSD and the initial dislocation density is less obvious for the range of initial dislocation densities modeled here due to the limited number of loading cycles reached.

Ultimately, the surface roughness evolution should be correlated with fatigue crack initiation, a process which typically occurs after thousands or millions of loading cycles if not more. The current study correlates the surface roughness evolution to the crystal size and initial dislocation density in the first few loading cycles. The main focus of this work is to give some insights on the physics of surface roughening. The method employed can be used to study the surface roughness evolution starting with any dislocation microstructure. However, in order to make the connection between the predicted surface roughening rates and fatigue failure, DDD simulations need to either simulate a large number of loading cycles, on the order of 1000 cycles at least, or alternatively, start with the dislocation microstructure and surface topology that typically evolve after that number of cycles. The first approach is unfeasible at this point because of the high computational demand of DDD simulations, which unlike other computational simulation methods like Molecular Dynamics or the Finite Element Method, have the distinctive feature of demanding more computational resources as the simulation proceeds since in most cases, the dislocation density tends to increase with increasing the number of loading cycles. The second approach however is more realistic. It requires the use of other experimental methods, such as Transmission Electron Microscopy (TEM) [55], or computational methods, such as Continuum Dislocation Dynamics (CDD) [56], to measure or predict the dislocation microstructure after the desired number of loading cycles and feeding them to the DDD simulation. The TEM method however works 
only for samples with smaller thicknesses and the CDD method does not resolve individual dislocations. Nevertheless, the results obtained from these methods can be fed into DDD pre-processors where a similar discrete dislocation microstructure can be generated. Atomic Force Microscopy (AFM) $[57,58]$ can be used to obtain surface roughness data, which can similarly be fed into DDD simulation codes as the initial surface geometry. In this hybrid approach, the DDD method serves as a way to resolve the fine mechanisms causing surface roughness evolution right before and at fatigue crack initiation since the low length and time scale mechanisms active at this critical point cannot be captured experimentally or modeled using field-based methods such as CDD. In this way, the entire fatigue process can be studied at the level of detail provided by the DDD method.

Another aspect of surface roughness evolution, which has not been addressed in this work is that of polycrystalline materials. While the surface roughness tracking method described above would not change in the case of modeling surface grains in polycrystalline materials, the DDD simulation itself should take into account the presence of grain boundaries by handling dislocation-grain boundary interactions. A number of works in the literatures report the use of two and three dimensional DDD in simulating the behavior of polycrystalline materials $[59,60,61,62,63,64]$.

\section{Summary and Conclusions}

In this work, three dimensional discrete dislocation dynamics simulations were performed to study the surface roughness evolution in FCC Ni single crystals subject to mechanical cyclic loading. Different crystal sizes starting with different initial dislocation densities were simulated. For the number of cycles modeled here, larger crystals showed a uniform surface step distribution compared to smaller crystals where the surface roughness was more localized in surface slip bands. The surface roughness was found to increase with the number of cycles and the roughening rate was found to be higher in larger crystals. A qualitative correlation between the dislocation microstructure evolution and surface roughness can be drawn where a higher and more spread out surface slip activity was found in the cases when the dislocation microstructure evolved to fill up the entire crystal volume. Surface cross-slip 
was found to play an important role in the thickening of surface slip bands. The maximum surface height evolution was predicted from the current to be proportional to the number of loading cycles and the square of the crystal size. The linear variation with the number of cycles implies a constant dislocation flux from the surface while the quadratic variation with crystal size is due to the increase of the number of sources per slip plane that contribute to the maximum height growth. The approach and results detailed here are a first step towards the development of fatigue crack nucleation models based on the surface stress and strain distributions.

\section{Acknowledgements}

This work was support by DARPA contract No. N6600112-14229, and a computing time Granted by XSEDE.

\section{References}

[1] R.R. Stephens R.I. Stephens, A. Fatemi and H.O. Fuchs. Metal Fatigue in Engineering, 2nd Ed. Wiley, 2000.

[2] J. Schijve. Fatigue damage in aircraft structures not wanted but tolerated. Int. J. Fatigue, 31:998-1011, 2009.

[3] H. Mughrabi. Fatigue, an everlasting materials problem - still en vogue. Procedia Eng., 2:3-26, 2010.

[4] S. Suresh. Fatigue of Materials, 2nd Ed. Cambridge University Press, 1998.

[5] J. Schijve. Fatigue of Structures and Materials, 2nd Ed. Springer, 2009.

[6] M.D. Sangid. The physics of fatigue crack initiation. Int. J. Fatigue, 57:58-72, 2013.

[7] H.J. Maier M.D. Sangid and H. Sehitoglu. The role of grain boundaries on fatigue crack initiation an energy approach. Int. J. Plasticity, 27(5):801-821, 2011.

[8] N. Kawagoishi Q.Y. Wang, C. Bathias and Q. Chen. Effect of inclusion on subsurface crack initiation and gigacycle fatigue strength. Int. J. Fatigue, 24(12):1269-1274, 2002.

[9] R. Gu and A.H.W. Ngan. The mechanisms of metal fatigue (ii). Phys. Stat. Sol. B., 47:359 - 396, 1971.

[10] J.A. Ewing and J.C.W. Humfrey. The fracture of metals under repeated alternations of stress. Phil. Trans. R. Soc. A, pages 241-250, 1903.

[11] U. Gosele U. Essmann and H. Mughrabi. A model of extrusions and intrusions in fatigued metals. I. point-defect production and growth of extrusions. Phil. Mag. A, 44:405-426, 1981. 
[12] U. Esmann K. Differt and H. Mughrabi. A model of extrusions and intrusions in fatigued metals II. surface roughening by random irreversible slip. Phil. Mag. A, 54(2):237-258, 1986.

[13] P. Lukas and L. Kunz. Role of persistent slip bands in fatigue. Phil. Mag., 84(3-5):317-330, 2004.

[14] J. Man, K. Obrtlik, and J. Polak. Extrusions and intrusions in fatigued metals. Part 1. state of the art and history. Phil. Mag., 89(16):1295-1336, 2009.

[15] M.E. Fine I.B. Kwon and J. Weertman. Microstructural studies on the initiation and growth of small fatigue cracks at 298, 77 and 4.2 K in polycrystalline copper. Acta Metall., 37(11):2927-2936, 1989.

[16] Z.S. Basinski and S.J. Basinski. Fundamental aspects of low amplitude cyclic deformation in facecentred cubic crystals. Prog. Mater. Sci., 36(9):89-148, 1992.

[17] H. Sehitoglu. Fatigue and Fracture, Thermal and Thermomechanical Fatigue of Structural Alloys, ASM Handbook. ASM Handbook, 1996.

[18] J. Man, K. Obrtlik, and J. Polak. Study of surface relief evolution in fatigued 316L austenitic stainless steel by AFM. Mat. Sci. Eng. A, 351(1-2):123-132, 2003.

[19] J. Man, M. Petrenec, K. Obrtlk, and J. Polak. AFM and TEM study of cyclic slip localization in fatigued ferritic X10CrAl24 stainless steel. Acta Mater., 52:5551-5561, 2004.

[20] T. Vystavel J. Polak, J. Man and M. Petrenec. The shape of extrusions and intrusions and initiation of stage I fatigue cracks. Mat. Sci. Eng. A, 517:204-211, 2009.

[21] M.D. Uchic, P.A. Shade, and D.M. Dimiduk. Plasticity of micrometer-scale single crystals in compression: a critical review. Annu. Rev. Mater. Res., 39(1):361-386, 2009.

[22] O. Kraft, P.A. Gruber, R. Monig, and D. Weygand. Plasticity in confined dimensions. Annu. Rev. Mater. Res., 40:293-317, 2010.

[23] D. Kiener, C. Motz, W. Grosinger, D. Weygand, and R. Pippan. Cyclic response of copper single crystal micro-beams. Scripta Mater., 63:500-503, 2010.

[24] C. Kirchlechner, P.J. Imrich, W. Liegl, J. Pornbacher, J.-S. Micha, O. Ulrich, and C. Motz. On the reversibility of dislocation slip during small scale low cycle fatigue. Acta Mater, 94:69 - 77, 2015.

[25] D. Liu, Y. He, D.J. Dunstan, B. Zhang, Z. Gan, P. Hu, and H. Ding. Anomalous plasticity in the cyclic torsion of micron scale metallic wires. Phys. Rev. Lett., 110(24), 2013.

[26] D. Liu, Y. He, L. Shen, J. Lei, S. Guo, and K. Peng. Accounting for the recoverable plasticity and size effect in the cyclic torsion of thin metallic wires using strain gradient plasticity. Mater. Sci. Eng. A, 647:84-90, 2015.

[27] J.A. El-Awady, H. Fan, and A.M. Hussein. Multiscale Modeling for Nanomechanics, chapter Discrete Dislocation Dynamics: Recent Advances in Modeling Size-Affected Plasticity. Springer Publishing, 2016.

[28] V.S. Deshpande, A. Needleman, and E. Van der Giessen. Discrete dislocation plasticity modeling of 
short cracks in single crystals. Acta Mater., 51:1-15, 2003.

[29] C. Déprés, C.F. Robertson, and M.C. Fivel. Low strain fatigue in 316L steel surface grains: a threedimension discrete dislocation dynamics modeling of the early cycles. part 1: Dislocation microstructures and mechanical behavior. Phil. Mag., 84(22):2257-2275, 2004.

[30] C. Déprés, C.F. Robertson, and M.C. Fivel. Low strain fatigue in 316L steel surface grains: a threedimension discrete dislocation dynamics modeling of the early cycles. part 2: Persistent slip markings and micro-crack nucleation. Phil. Mag., 86(1):79-97, 2006.

[31] J.A. El-Awady, N.M. Ghoniem, and H. Mughrabi. Dislocation modelling of localized plasticity in persistant slip bands. In B.L. Adams and A. Garmestani, editors, Proceedings of the 136th TMS Annual Meeting and Exhibition, pages 23-35, 2007.

[32] A.M. Hussein and J.A. El-Awady. Quantifying dislocation microstructure evolution and cyclic hardening in fatigued nickel single-crystals. Submitted, 2015.

[33] M. Tang M. Rhee T. Oppelstrup G. Hommes T. G. Pierce A. Arsenlis, W. Cai and V. V. Bulatov. Enabling strain hardening simulations with dislocation dynamics. Mod. Simul. Mater. Sci. Eng., $15(6): 553-595,2007$.

[34] A.M. Hussein, S.I. Rao, M.D. Uchic, D.M. Dimiduk, and J.A. El-Awady. Microstructurally based cross-slip mechanisms and their effects on dislocation microstructure evolution in fcc crystals. Acta Mater., 85:180-190, 2015.

[35] S.I. Rao, D.M. Dimiduk, J.A. El-Awady, T.A. Parthasarathy, M.D. Uchic, and C. Woodward. Atomistic simulations of athermal cross-slip nucleation at screw dislocation intersections in face-centered cubic nickel. Phil. Mag., 89(34-36):3351-3369, 2009.

[36] S.I. Rao, D.M. Dimiduk, J.A. El-Awady, T.A. Parthasarathy, M.D. Uchic, and C. Woodward. Activated states for cross-slip at screw dislocation intersections in face-centered cubic nickel and copper via atomistic simulation. Acta Mater., 58:5547-5557, 2010.

[37] S.I. Rao, D.M. Dimiduk, T.A. Parthasarathy, J. El-Awady, Woodward C., and M.D. Uchic. Calculations of intersection cross-slip activation energies in fcc metals using nudged elastic band method. Acta Mater., 59(19):7135 - 7144, 2011.

[38] S.I. Rao, D.M. Dimiduk, J.A. El-Awady, T.A. Parthasarathy, M.D. Uchic, and C. Woodward. Spontaneous athermal cross-slip nucleation at screw dislocation intersections in fcc metals and L12 intermetallics investigated via atomistic simulations. Phil. Mag., 93(22):3012-3028, 2013.

[39] S.I. Rao, D.M. Dimiduk, T.A. Parthasarathy, M.D. Uchic, and C. Woodward. Atomistic simulations of surface cross-slip nucleation in face-centered cubic nickel and copper. Acta Mater., 61(7):2500-2508, 2013.

[40] R. DeWit. The continuum theory of stationary dislocations. Solid State Phys., 10:249-292, 1960. 
[41] D. Weygand, L. H. Friedman, E. Van der Giessen, and A. Needleman. Aspects of boundary-value problem solutions with three-dimensional dislocation dynamics. Mod. Simul. Mater. Sci. Eng., 10:437468, 2002.

[42] C. Déprés, M. Fivel, Ch. Robertson, A. Fissolo, and M. Verdier. etude des stades precurseurs de l'endommagement en fatigue : experiences et simulations a l'echelle des dislocations. J. Phys. IV France, 106:81-90, 2003.

[43] M. Peach and J.S. Koehler. The forces exerted on dislocations and the stress fields produced by them. Phys. Rev., 80(3):436, 1950.

[44] R. Deltombe, K.J. Kubiak, and M. Bigerelle. How to select the most relevant 3d roughness parameters of a surface. Scanning, 36:150-160, 2014.

[45] B. Mandelbrot. The Fractal Geometry of Nature. Freeman: San Francisco, 1982.

[46] C.D. Zachmann and J. Brickmann. Hausdorff dimension as a quantification of local roughness of protein surfaces. J. Chem. Inf. Comut. Sci., 32:120-122, 1992.

[47] J. M. Elson and J.M. Bennett. Calculation of the power spectral density from surface profile data. Appl. Optics, 34(1):201-208, 1995.

[48] A. Duparre, J.F. Borrull, S. Gliech, G. Notni, J. Steinert, and J.M. Bennett. Surface characterization techniques for determining the root-mean-square roughness and power spectral densities of optical components. Appl. Optics, 41(1):154-171, 2002.

[49] J.A. El-Awady, S.B. Biner, and N.M. Ghoniem. A self-consistent boundary element, parametric dislocation dynamics formulation of plastic flow in finite volumes. J. Mech. Phys. Solids, 56(5):2019-2035, 2008.

[50] J.T. Fourie. The surface effect in micro strain and cyclic loading. Script. Metall., 2(1):63-64, 1968.

[51] J.T. Fourie. The flow stress gradient between the surface and centre of deformed copper single crystals. Phil. Mag., 17(146):735-756, 1968.

[52] H. Mughrabi. Investigations of plastically deformed copper single crystals in the stress-applied state. i. a study of the dislocation behaviour in the surface region and in the bulk. Phys. Status Solidi, 39:317-327, 1970.

[53] J.P. Hirth and J. Lothe. Theory of dislocations. John Wiley \& Sons, New York, 2nd ed. edition, 1982.

[54] D. Hull and D.J. Bacon. Introduction to Dislocations. Butterworth-Heinemann, 2001.

[55] J. M. Howe B. Fultz. Transmission Electron Microscopy and Diffractometry of Materials. Springer, 2012.

[56] T. Hochrainer, S. Sanfeld, M. Zaiser, and P. Gumbsch. Continuum dislocation dynamics: Towards a physical theory of crystal plasticity. J. Mech. Phys. Solid., 63:167-178, 2014.

[57] L. Cretegny and A. Saxena. Afm characterization of the evolution of surface deformation during fatigue 
in polycrystalline copper. Acta Mater., 49:3755-3765, 2001.

[58] L. Cretegny and A. Saxena. Evolution of surface deformation during fatigue of ph13-8 stainless steel using atomic force microscopy. Fatigue and Fracture of Engineering Materials and Structures, 25:305$314,2002$.

[59] B. Liu, D. Raabe, P. Eisenlohr, F. Roters, A. Arsenlis, and G. Hommes. Dislocation interactions and low-angle grain boundary strengthening. Acta Mater., 59(7125-7134), 2011.

[60] B. Liu, P. Eisenlohr P., F. Roters, and D. Raabe. Simulation of dislocation penetration through a general low-angle grain boundary. Acta Mater., 60(5380-5390), 2012.

[61] C. Zhou and R. Lesar. Dislocation dynamics simulations of plasticity in polycrystalline thin films. Int. J. Plasticity, 30-31:185-201, 2012.

[62] S.S. Quek, Z. Wu, Y.W. Zhang, and D. Srolovitz. Polycrystal deformation in a discrete dislocation dynamics framework. Acta Mater., 75:92-105, 2014.

[63] H. Fan, S. Aubry, A. Arsenlis, and J. El-Awady. The role of twinning deformation on the hardening response of polycrystalline magnesium from discrete dislocation dynamics simulations. Acta Mater., 92:126-139, 2015.

[64] H. Fan, S. Aubry, A. Arsenlis, and J. El-Awady. Orientation influence on grain size effects in ultrafinegrained magnesium. Scripta Mater., 97:25-28, 2015. 


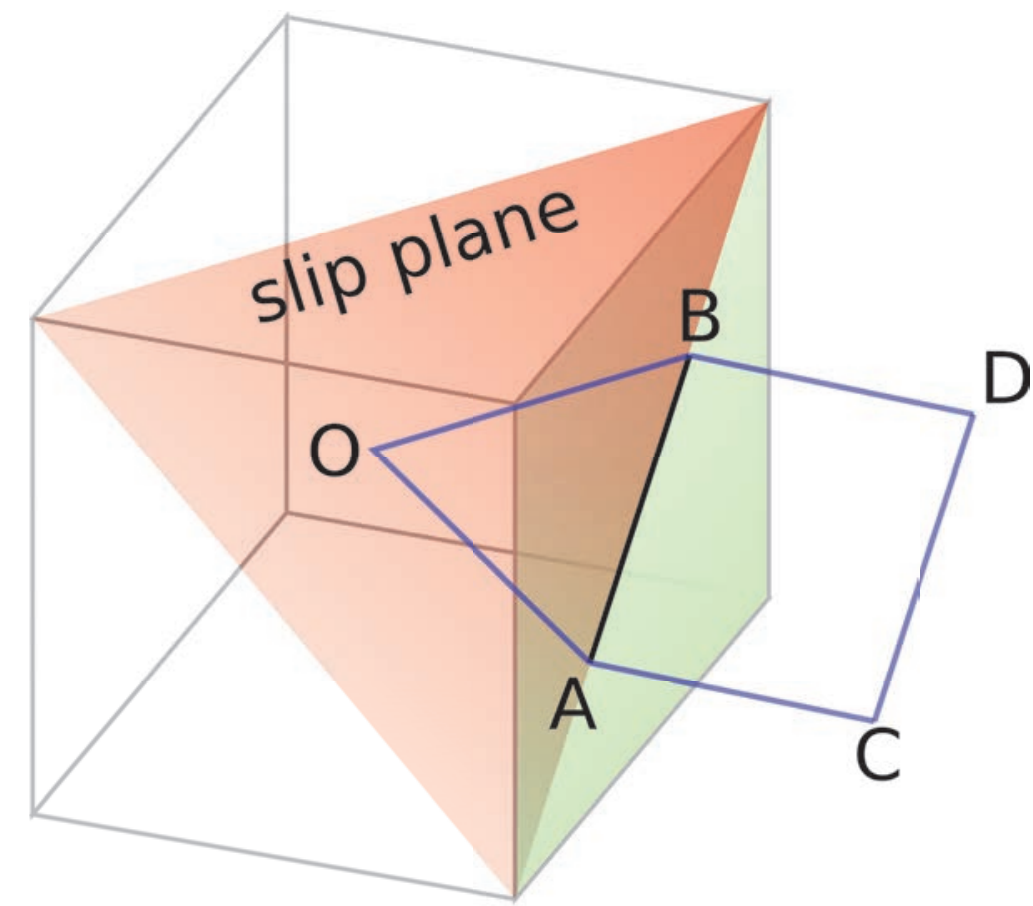

Figure 1: A schematic showing a dislocation $(A B)$ that escaped the free surface in black and the equivalent dislocation loop anchored at point $\mathrm{O}$ in blue. The dislocation slip plane is shown in green and the crystal surface is shown in red. 


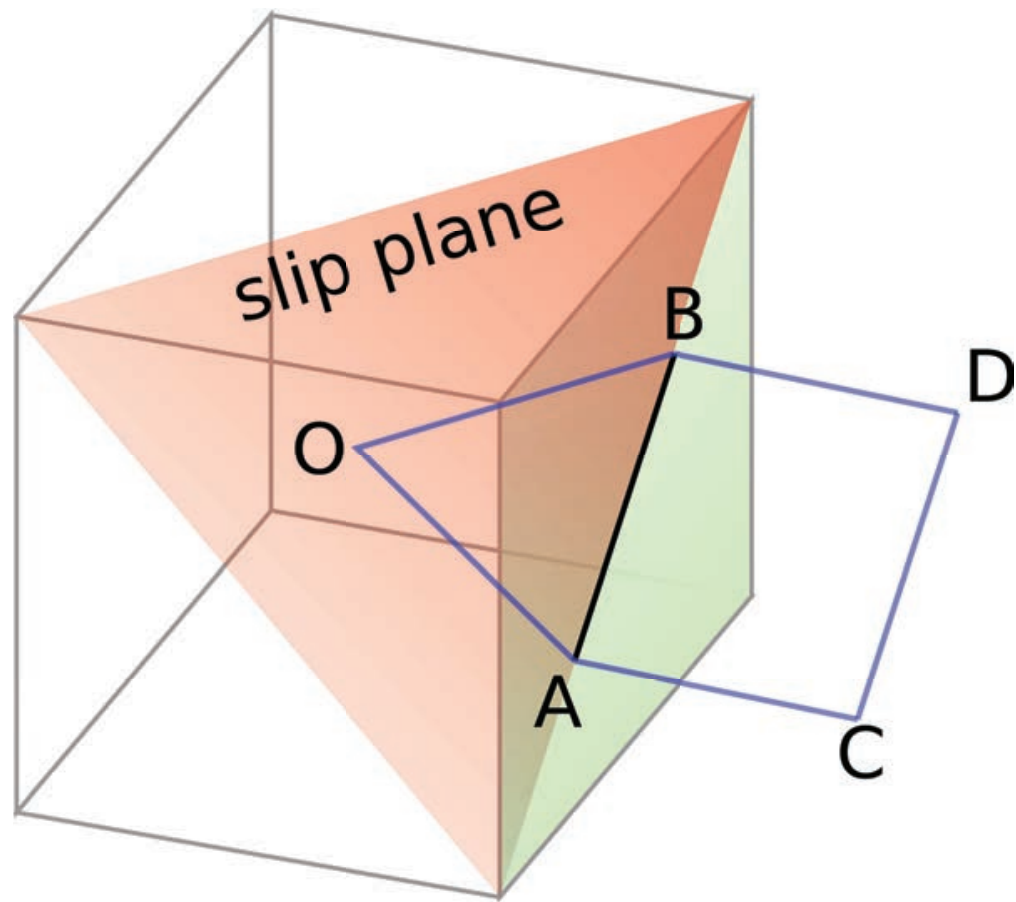

Figure 1: A schematic showing a dislocation $(A B)$ that escaped the free surface in black and the equivalent dislocation loop anchored at point $\mathrm{O}$ in blue. The dislocation slip plane is shown in green and the crystal surface is shown in red. 


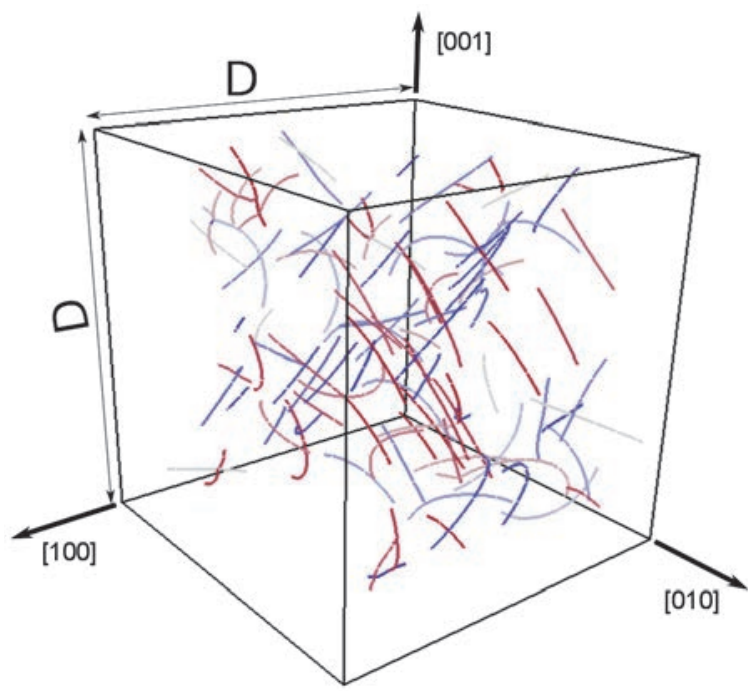

Figure 2: Schematic of the simulation cell showing the initial dislocation microstrucutre. The colors represent dislocation belonging to different slip systems. The loading is imposed along the [001] direction 


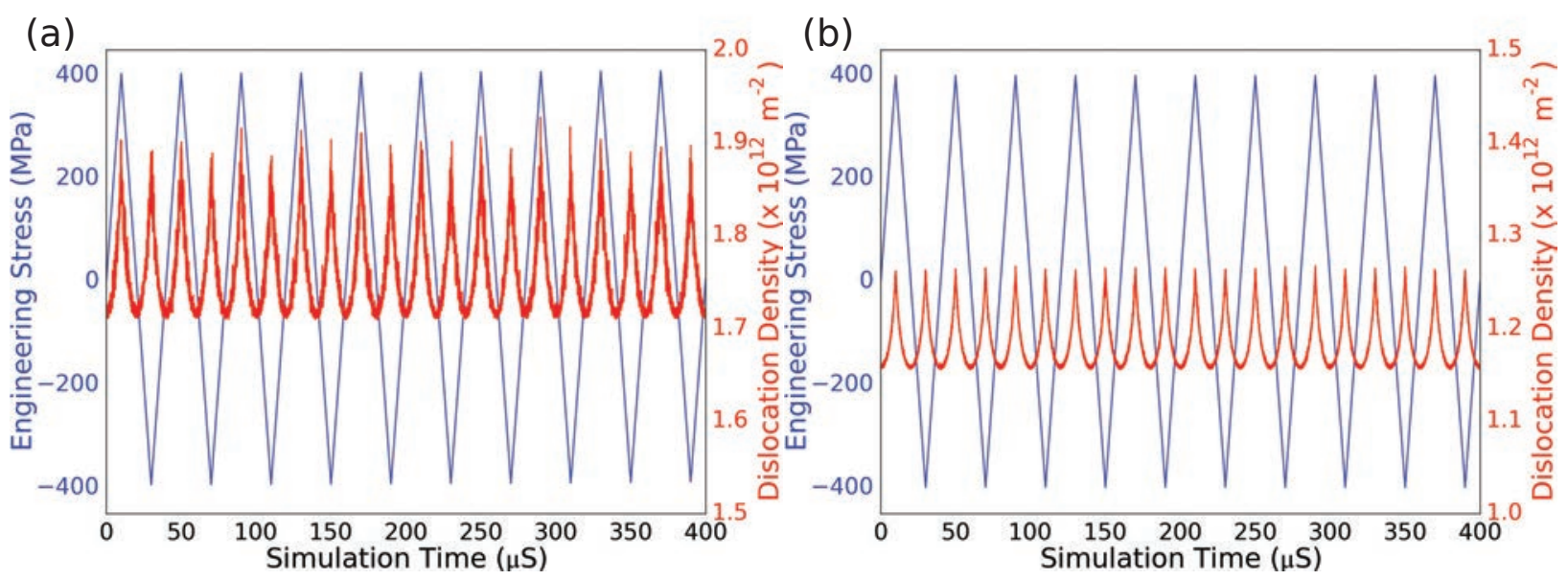

(c)

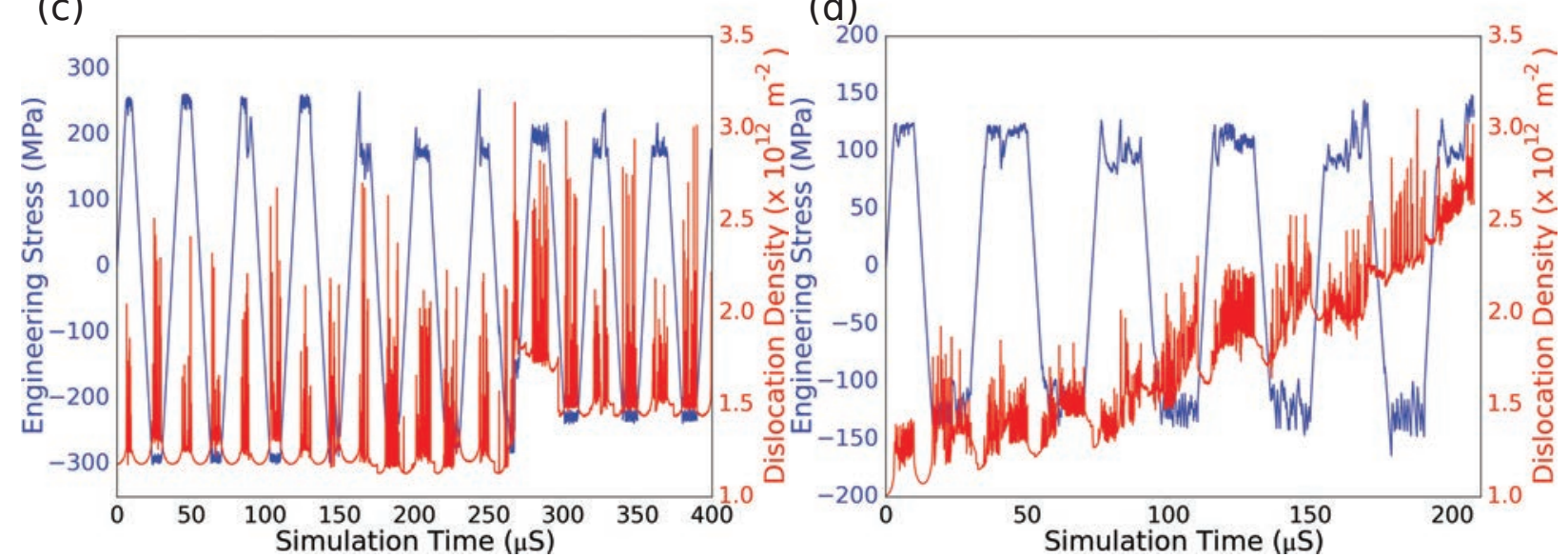

(e)

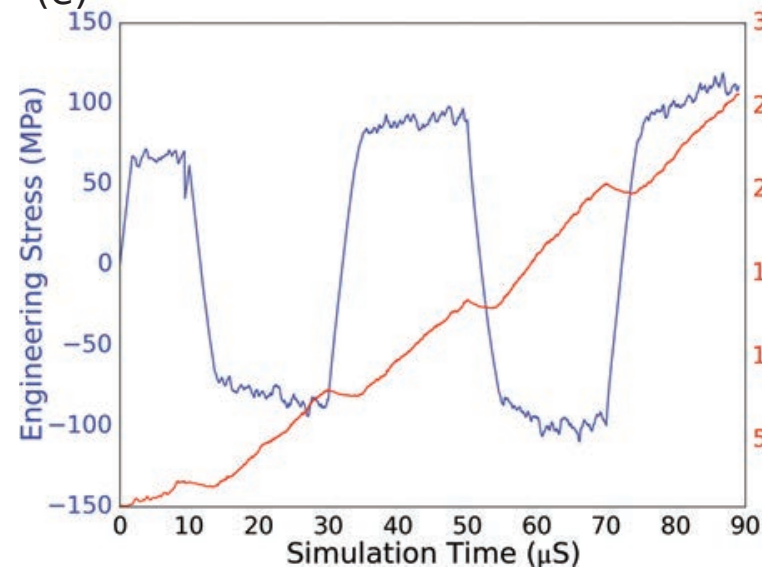

(f)

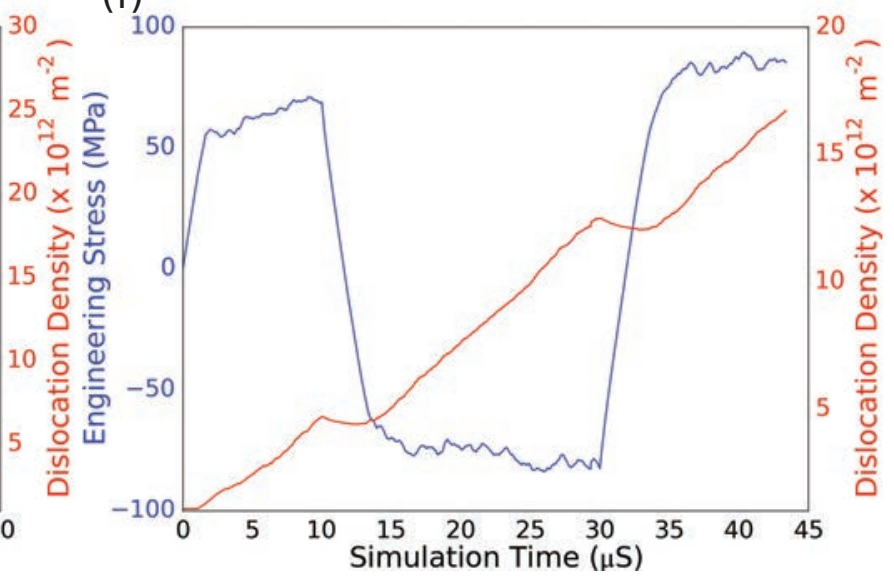

Figure 3: The engineering stress and dislocation density as a function of simulation time for simulations with an initial dislocation density of $\rho_{o}=10^{12} \mathrm{~m}^{-2}$ and crystal sizes: (a) $0.5 \mu \mathrm{m}$; (b) $0.75 \mu \mathrm{m}$; (c) $1.0 \mu \mathrm{m}$; (d) $2.0 \mu \mathrm{m}$; (e) $5.0 \mu \mathrm{m}$; and (f) $7.5 \mu \mathrm{m}$. 

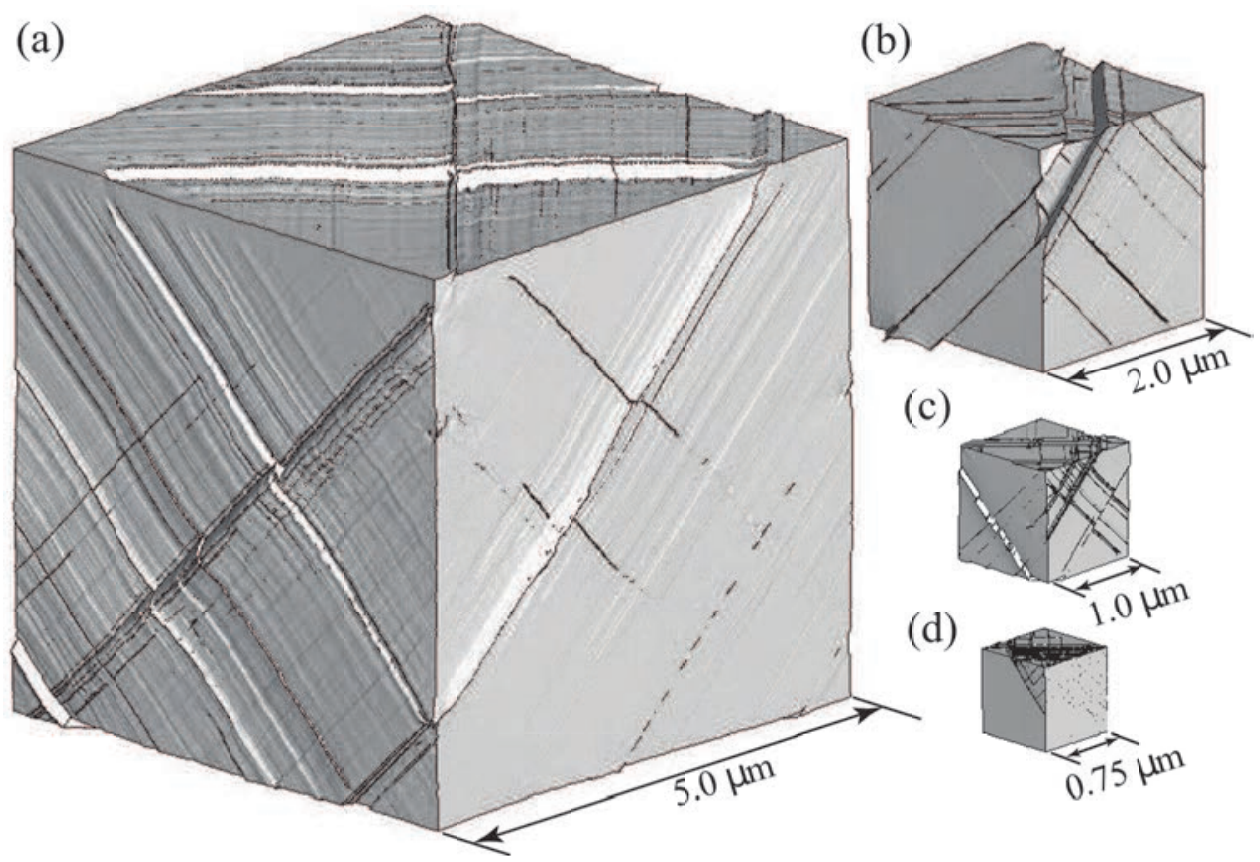

(c)

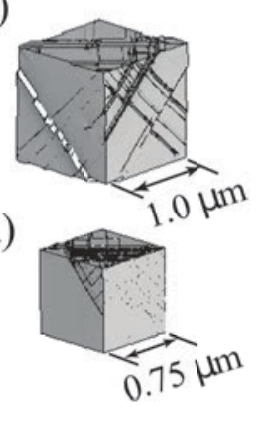

Figure 4: Reconstructions of the crystal surface morphology after 5 loading cycles for crystals having: (a) $D=5.0 \mu \mathrm{m}$ and $\rho_{o}=10^{11} \mathrm{~m}^{-2}$; (b) $D=2.0 \mu \mathrm{m}$ and $\rho_{o}=10^{12} \mathrm{~m}^{-2}$; (c) $D=1.0 \mu \mathrm{m}$ and $\rho_{o}=10^{13} \mathrm{~m}^{-2}$; and (d) $D=0.75 \mu \mathrm{m}$ and $\rho_{o}=10^{12} \mathrm{~m}^{-2}$. The surface displacement is magnified 10 times. 

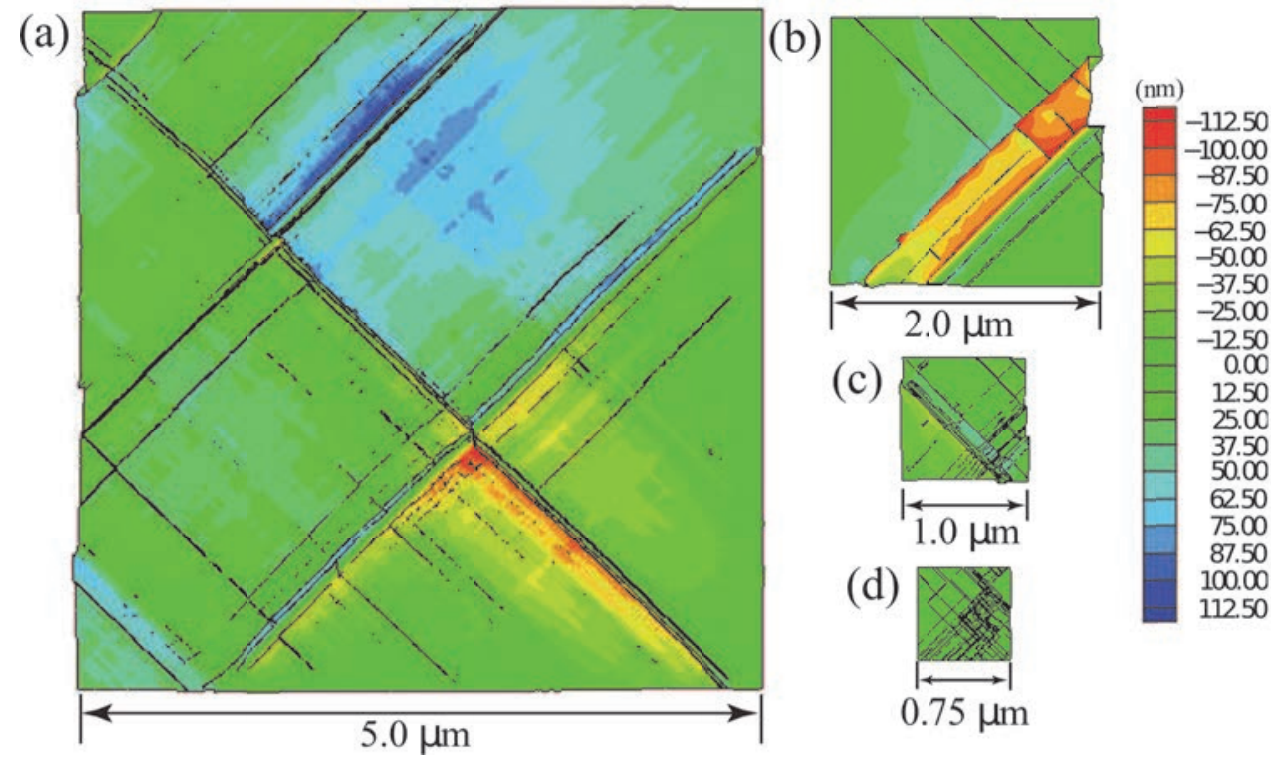

Figure 5: The displacement contours of the top surface after 5 loading cycles for crystals having: (a) $D=5.0$ $\mu \mathrm{m}$ and $\rho_{o}=10^{11} \mathrm{~m}^{-2}$; (b) $D=2.0 \mu \mathrm{m}$ and $\rho_{o}=10^{12} \mathrm{~m}^{-2}$; (c) $D=1.0 \mu \mathrm{m}$ and $\rho_{o}=10^{13} \mathrm{~m}^{-2}$; and (d) $D=0.75 \mu \mathrm{m}$ and $\rho_{o}=10^{12} \mathrm{~m}^{-2}$. 


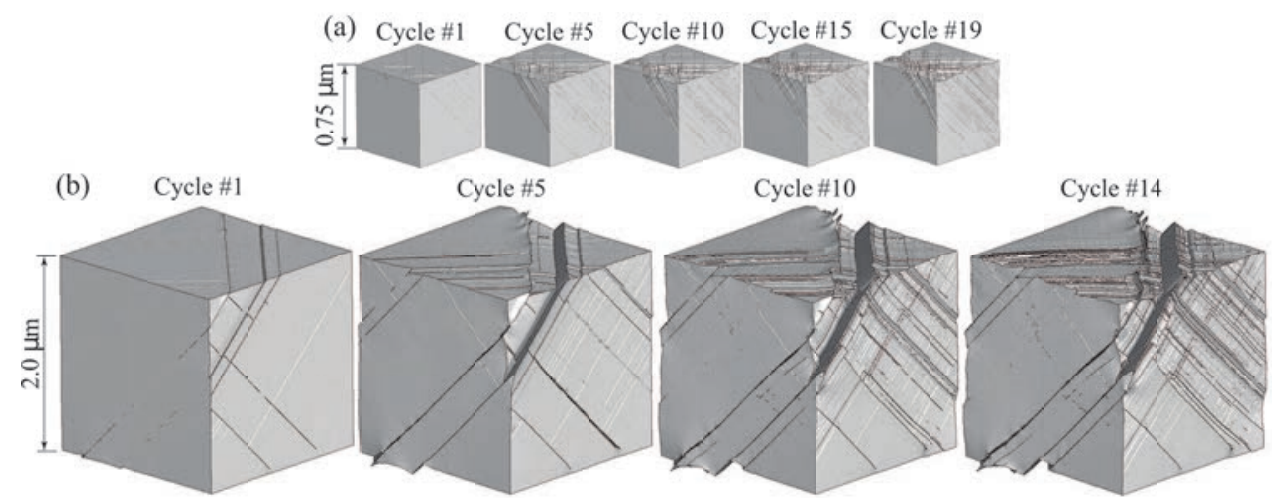

Figure 6: The evolution of surface displacements with the number of loading cycles for crystals having the same initial dislocation density of $\rho_{o}=10^{12} \mathrm{~m}^{-2}$ and sizes: (a) $D=0.75 \mu \mathrm{m}$; and (b) $D=2.0 \mu \mathrm{m}$. 
a)

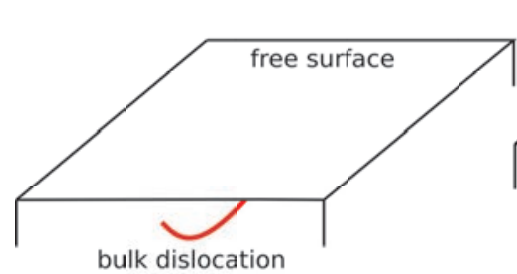

c)

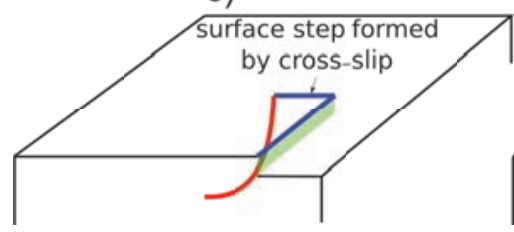

b)

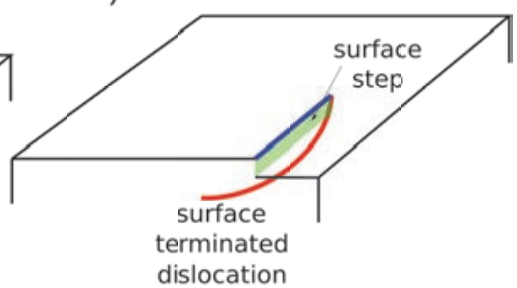

d)

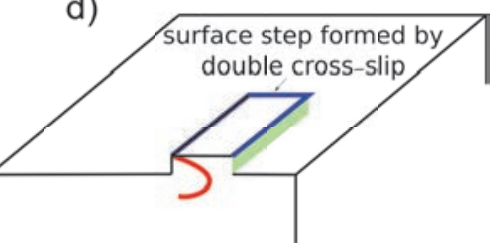

Figure 7: Schematic of the formation of surface slip bands through double surface cross-slip: (a) A dislocation (red) residing inside the crystal approaches the crystal surface. (b) A surface step (green) forms as the dislocation zips on the surface. (c) The dislocation intersecting the free surface cross-slips and moves on a different slip plane. (d) The dislocation undergoes another cross-slip event, which takes it back to a plane parallel to its original glide plane. As the load is reversed it glides in the opposite direction and deposites a second surface (blue) step on the crystal surface. The glide of the dislocation in the opposite directions causes the volume of the crystal bounded by the original glide plane and the current glide plane to extrude or intrude according to the sign of the Burgers vector. 

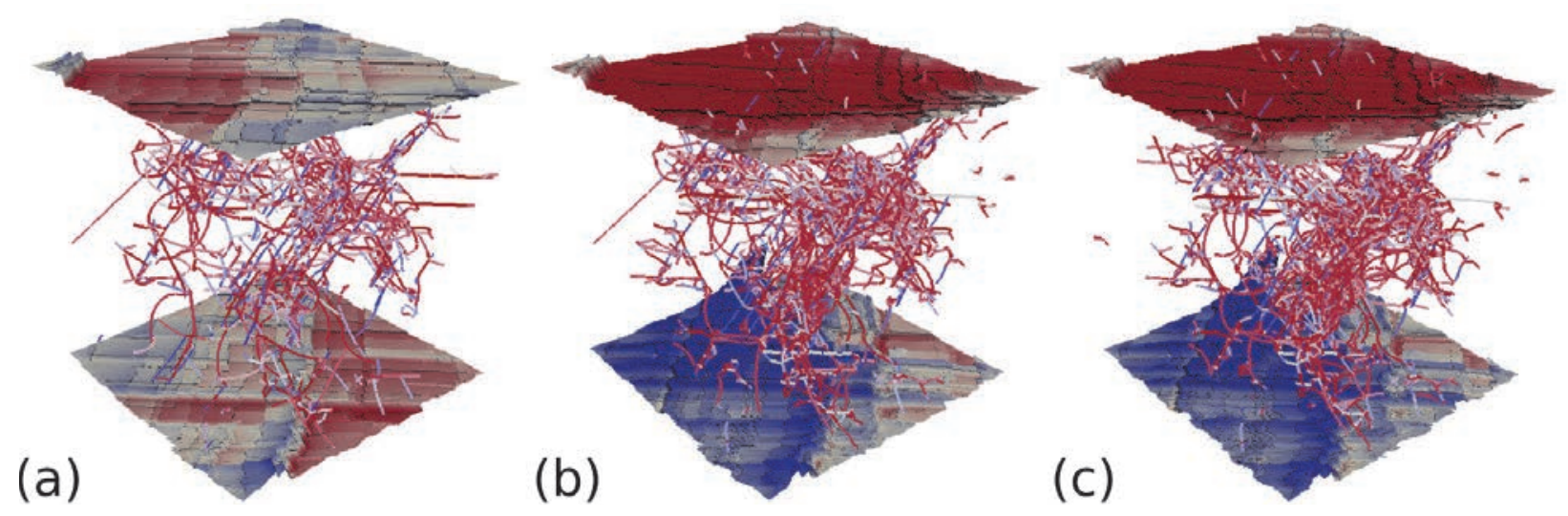

Figure 8: The dislocation microstructure and surface roughness evolution in a $D=2.0 \mu \mathrm{m}$ crystal having an initial dislocation density of $\rho_{o}=10^{13} \mathrm{~m}^{-2}$, after: (a) 2; (b) 3; and (c) 5 loading cycles. The colors represents the contours of the normal displacement field and the dislocations are colored according to their slip system. 

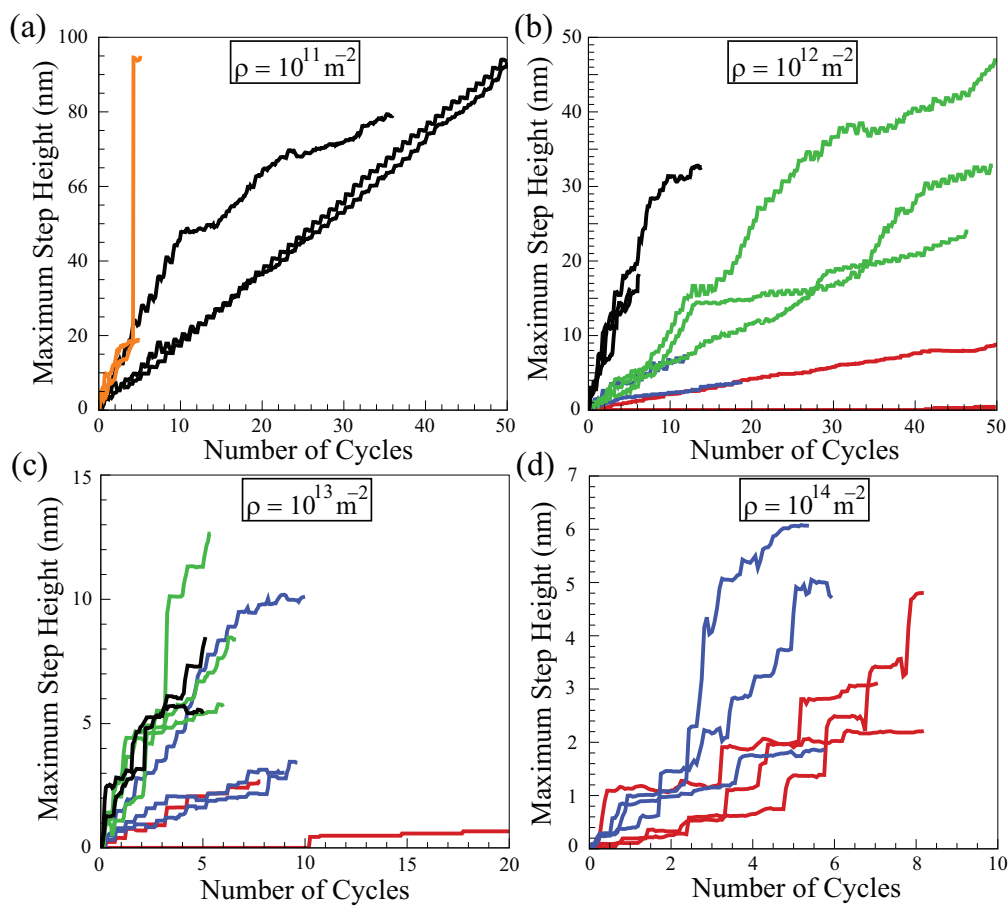

Figure 9: The maximum surface height versus the number of cycles for different crystal sizes at a fixed dislocation density: (a) $\rho_{o}=10^{11} \mathrm{~m}^{-2}$; (b) $\rho_{o}=10^{12} \mathrm{~m}^{-2}$; (c) $\rho_{o}=10^{13} \mathrm{~m}^{-2}$; and (d) $\rho_{o}=10^{14} \mathrm{~m}^{-2}$. The red, blue, green, black, and orange curves are for crystal sizes of $D=0.5,0.75,1.0,2.0$, and $5.0 \mu \mathrm{m}$, respectively. See online version for colors. 

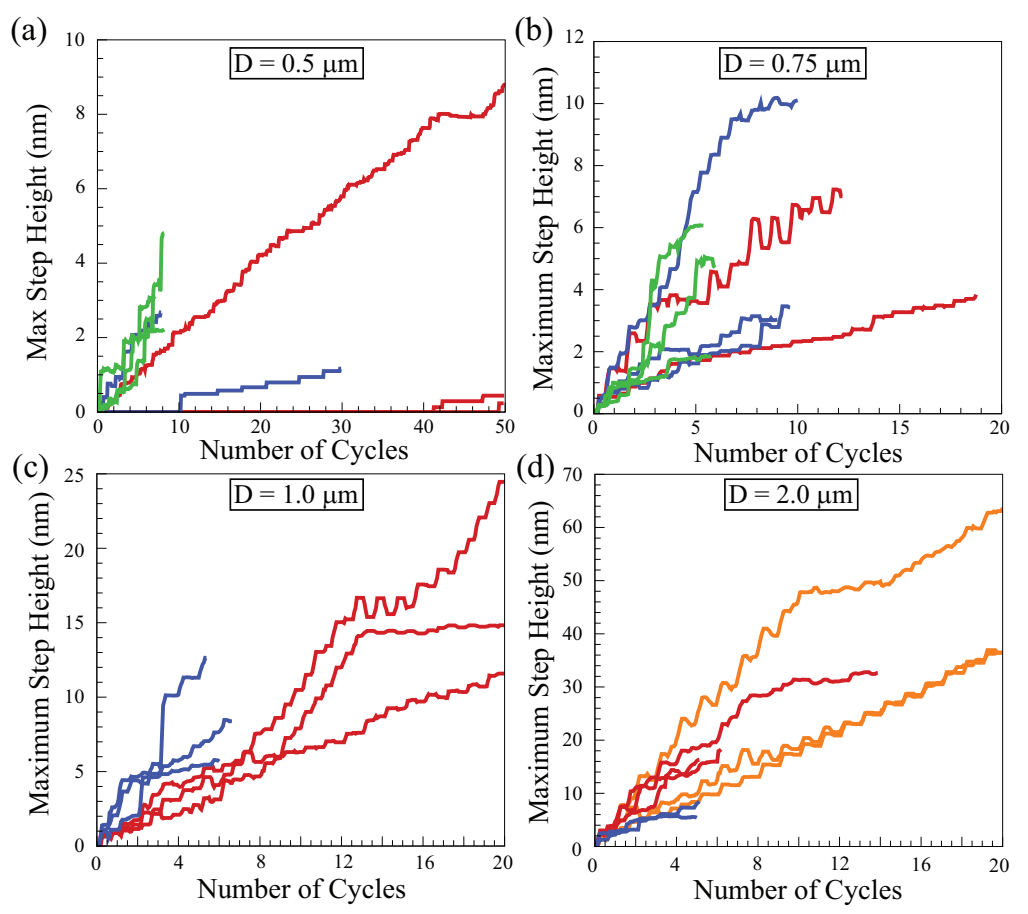

Figure 10: The maximum surface height versus the number of cycles for different initial dislocation densities at a fixed crystal size: (a) $D=0.5 \mu \mathrm{m}$; (b) $D=0.75 \mu \mathrm{m}$; (c) $D=1.0 \mu \mathrm{m}$; and (d) $D=2.0 \mu \mathrm{m}$. The orange, red, blue, and green curves are for initial dislocation densities of $\rho_{o}=10^{11}, 10^{12}, 10^{13}$, and $10^{14} \mathrm{~m}^{-2}$, respectively. See online version for colors. 
(a)

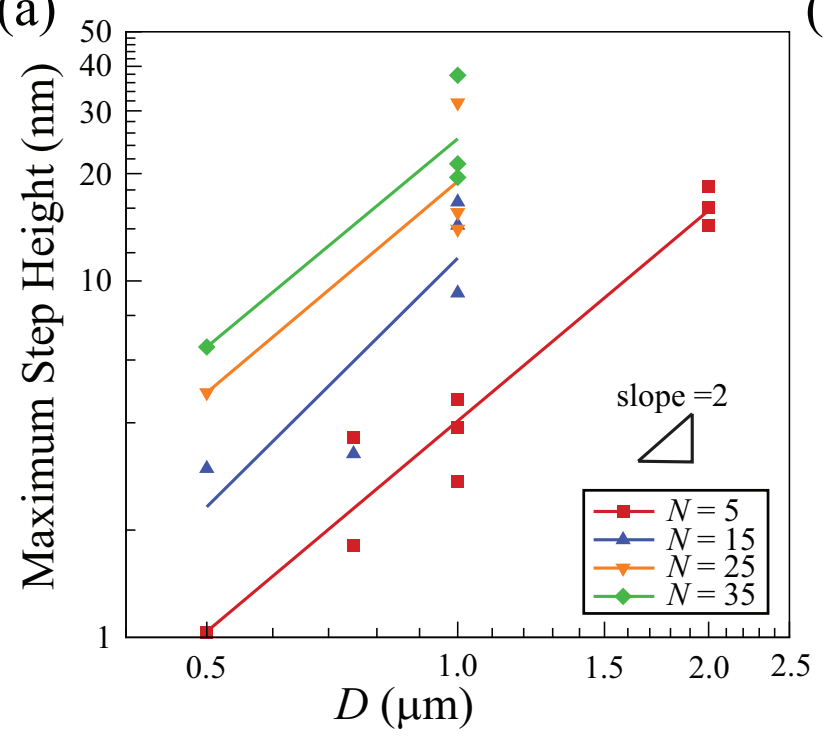

(b)

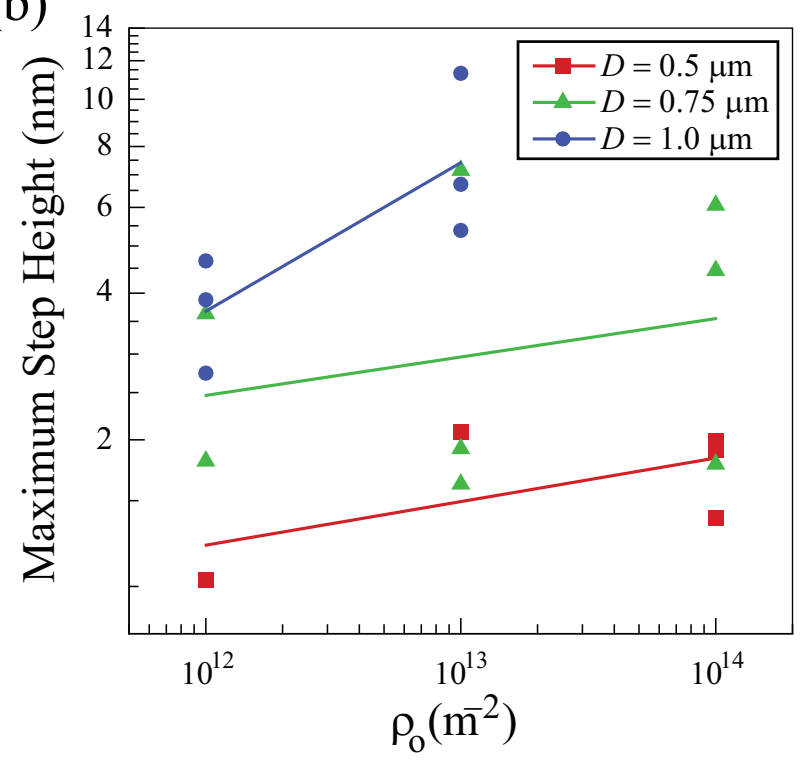

Figure 11: (a) The maximum surface height as a function of crystal size for different numbers of loading cycles and a fixed initial dislocation density of $\rho_{o}=10^{12} \mathrm{~m}^{-2}$; and (b) The maximum surface height as a function of the initial dislocation density for different crystal sizes after 5 loading cycles. 

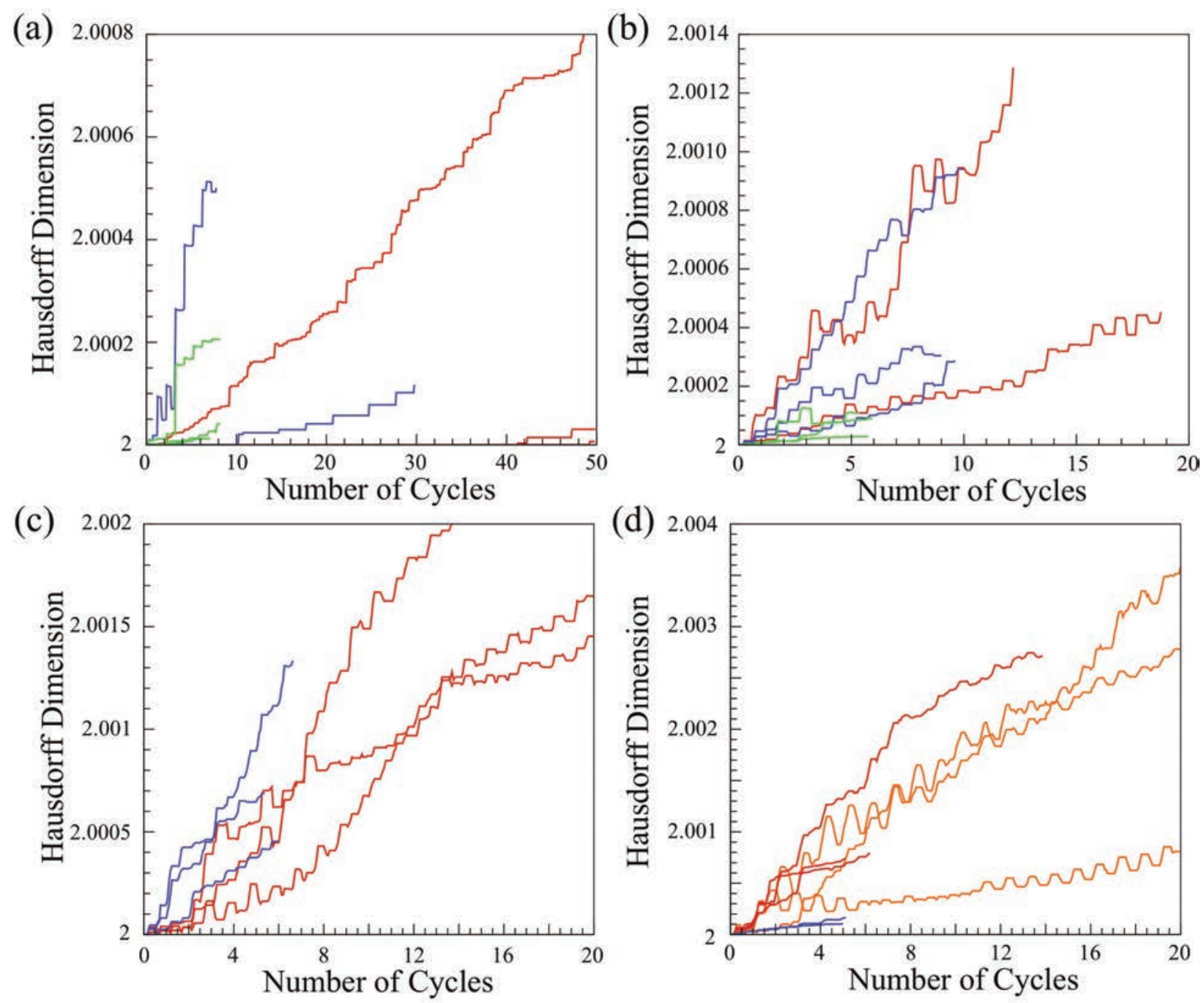

Figure 12: The Hausdorff dimension versus the number of loading cycles for different initial densities at a fixed crystal size of: (a) $D=0.5 \mu \mathrm{m}$; (b) $D=0.75 \mu \mathrm{m}$; (c) $D=1.0 \mu \mathrm{m}$; and (d) $D=2.0 \mu \mathrm{m}$. The orange, red, blue, and green curves are for initial dislocation densities of $\rho_{o}=10^{11}, 10^{12}, 10^{13}$, and $10^{14} \mathrm{~m}^{-2}$, respectively. See online version for colors. 

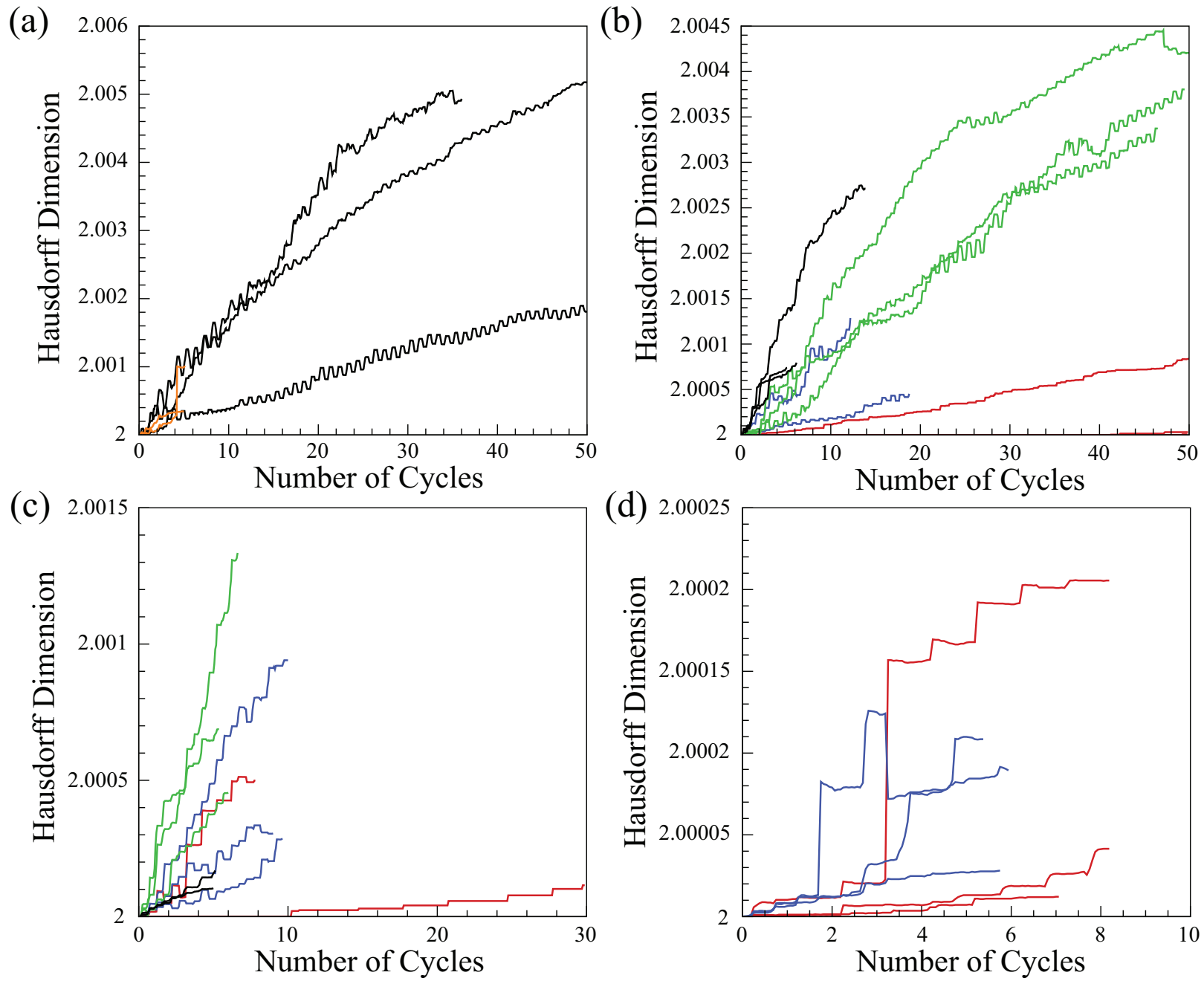

Figure 13: The Hausdorff dimension versus the number of loading cycles for different crystal sizes and fixed initial dislocation density of: (a) $\rho_{o}=10^{11} \mathrm{~m}^{-2}$; (b) $\rho_{o}=10^{12} \mathrm{~m}^{-2}$; (c) $\rho_{o}=10^{13} \mathrm{~m}^{-2}$; and (d) $\rho_{o}=10^{14}$ $\mathrm{m}^{-2}$. The red, blue, green, black, and orange curves are for crystal sizes of $D=0.5,0.75,1.0,2.0$, and 5.0 $\mu \mathrm{m}$, respectively. See online version for colors. 


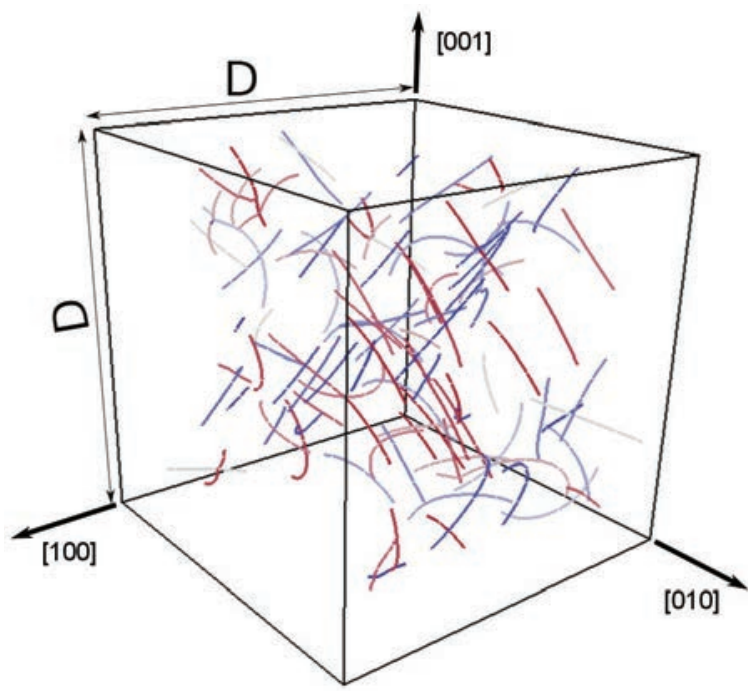

Figure 2: Schematic of the simulation cell showing the initial dislocation microstrucutre. The colors represent dislocation belonging to different slip systems. The loading is imposed along the [001] direction 

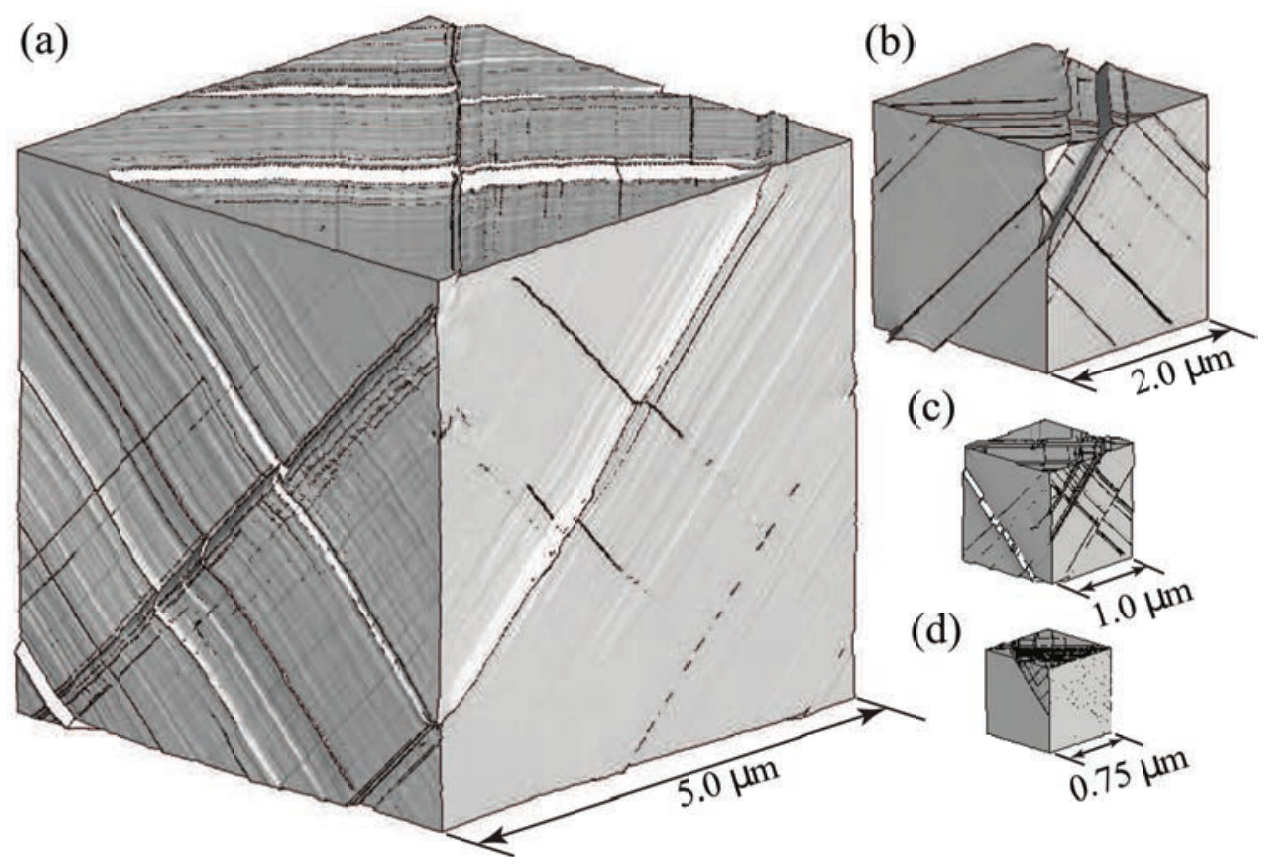

(c)

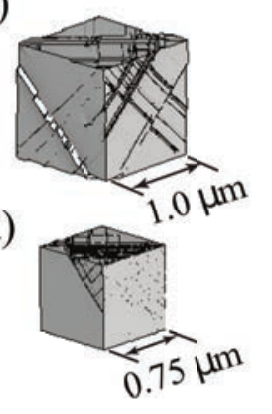

Figure 4: Reconstructions of the crystal surface morphology after 5 loading cycles for crystals having: (a) $D=5.0 \mu \mathrm{m}$ and $\rho_{o}=10^{11} \mathrm{~m}^{-2}$; (b) $D=2.0 \mu \mathrm{m}$ and $\rho_{o}=10^{12} \mathrm{~m}^{-2}$; (c) $D=1.0 \mu \mathrm{m}$ and $\rho_{o}=10^{13} \mathrm{~m}^{-2}$; and (d) $D=0.75 \mu \mathrm{m}$ and $\rho_{o}=10^{12} \mathrm{~m}^{-2}$. The surface displacement is magnified 10 times. 

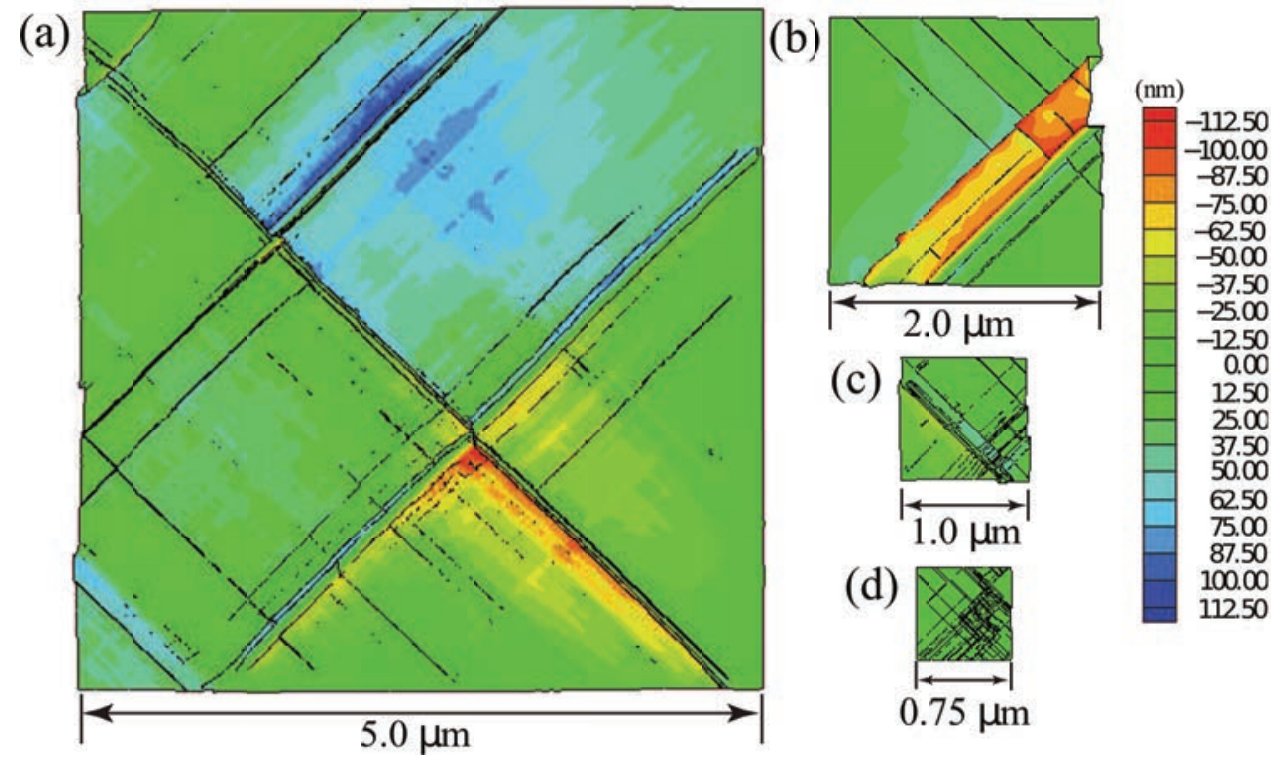

Figure 5: The displacement contours of the top surface after 5 loading cycles for crystals having: (a) $D=5.0$ $\mu \mathrm{m}$ and $\rho_{o}=10^{11} \mathrm{~m}^{-2}$; (b) $D=2.0 \mu \mathrm{m}$ and $\rho_{o}=10^{12} \mathrm{~m}^{-2}$; (c) $D=1.0 \mu \mathrm{m}$ and $\rho_{o}=10^{13} \mathrm{~m}^{-2}$; and (d) $D=0.75 \mu \mathrm{m}$ and $\rho_{o}=10^{12} \mathrm{~m}^{-2}$. 


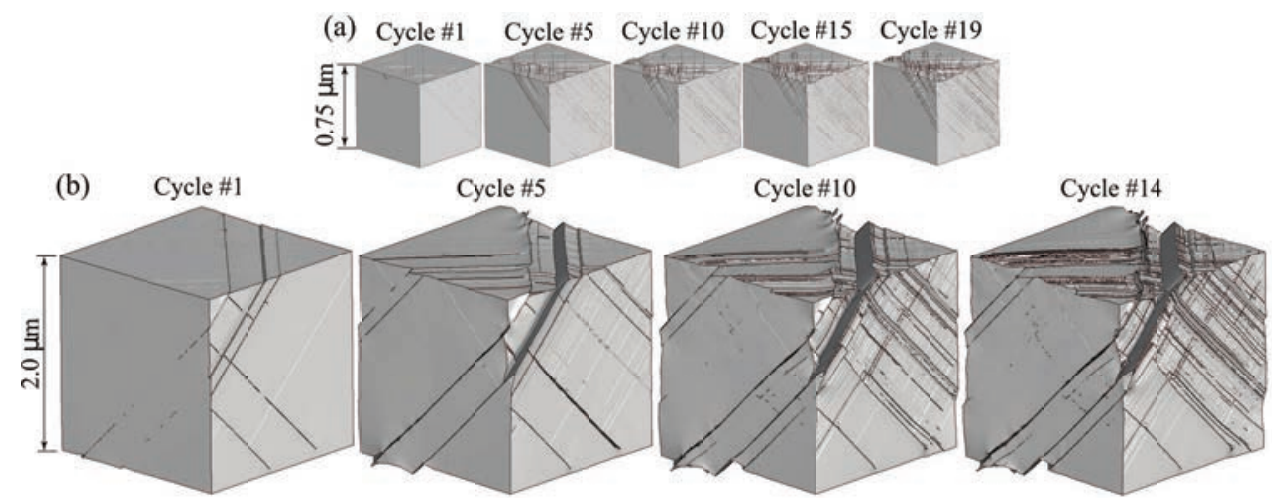

Figure 6: The evolution of surface displacements with the number of loading cycles for crystals having the same initial dislocation density of $\rho_{o}=10^{12} \mathrm{~m}^{-2}$ and sizes: (a) $D=0.75 \mu \mathrm{m}$; and (b) $D=2.0 \mu \mathrm{m}$. 
a)

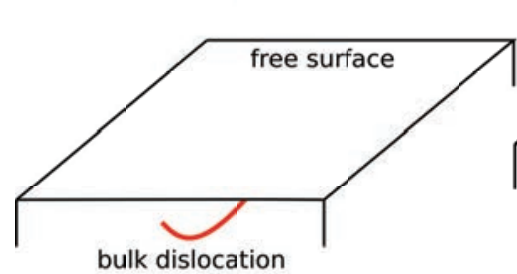

c)

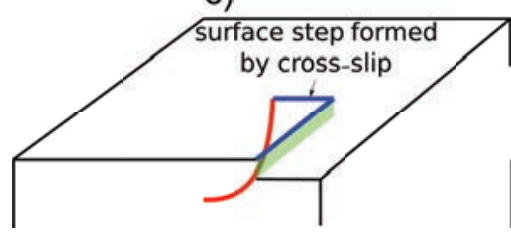

b)

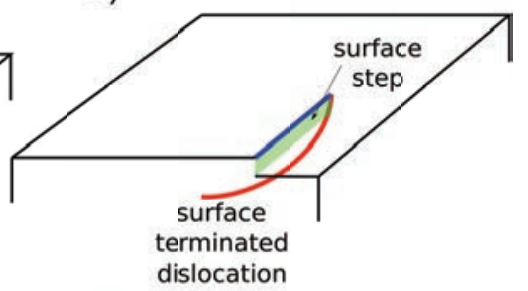

d)

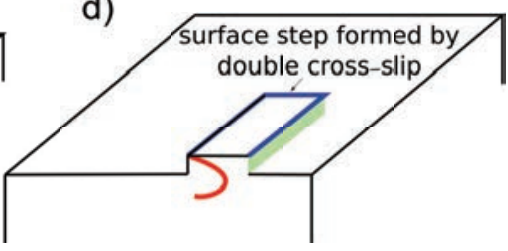

Figure 7: Schematic of the formation of surface slip bands through double surface cross-slip: (a) A dislocation (red) residing inside the crystal approaches the crystal surface. (b) A surface step (green) forms as the dislocation zips on the surface. (c) The dislocation intersecting the free surface cross-slips and moves on a different slip plane. (d) The dislocation undergoes another cross-slip event, which takes it back to a plane parallel to its original glide plane. As the load is reversed it glides in the opposite direction and deposites a second surface (blue) step on the crystal surface. The glide of the dislocation in the opposite directions causes the volume of the crystal bounded by the original glide plane and the current glide plane to extrude or intrude according to the sign of the Burgers vector. 

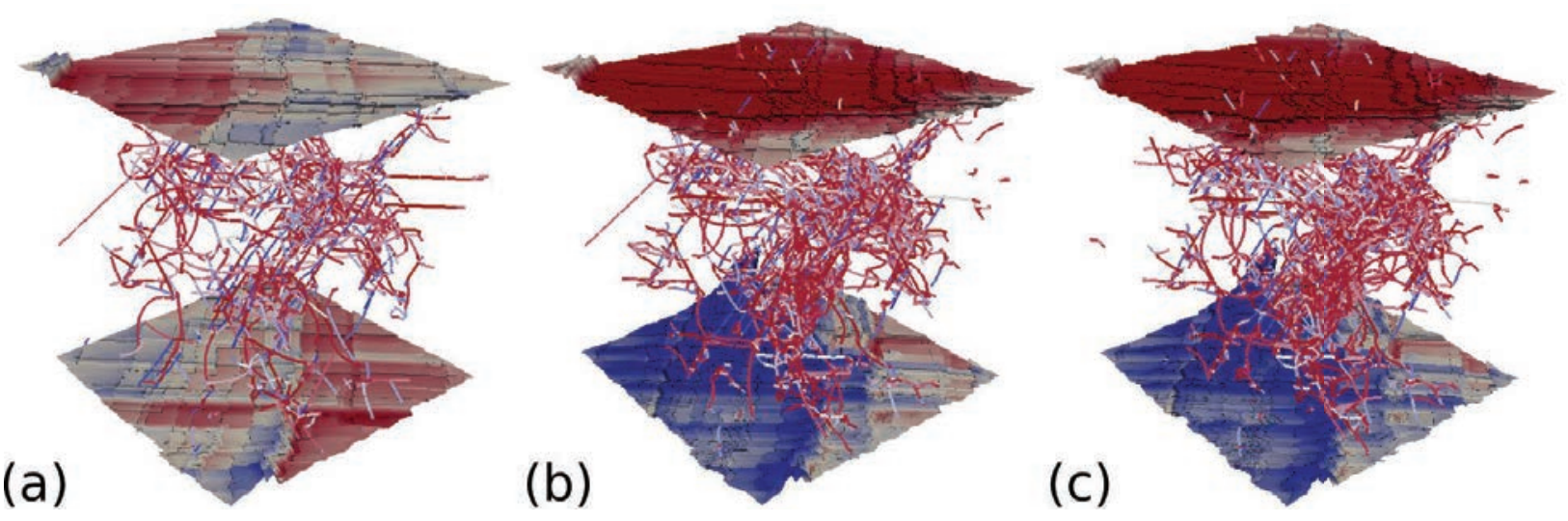

Figure 8: The dislocation microstructure and surface roughness evolution in a $D=2.0 \mu \mathrm{m}$ crystal having an initial dislocation density of $\rho_{o}=10^{13} \mathrm{~m}^{-2}$, after: (a) 2; (b) 3; and (c) 5 loading cycles. The colors represents the contours of the normal displacement field and the dislocations are colored according to their slip system. 